\title{
Correcting wavefield gradients for the effects of local small-scale heterogeneities
}

\author{
Sneha Singh ${ }^{1}$, Yann Capdeville ${ }^{1}$, Heiner Igel ${ }^{2}$ \\ ${ }^{1}$ Laboratoire de Planétologie et de Géodynamique, UMR CNRS 6112, Université de Nantes, \\ 44300 Nantes, France. \\ ${ }^{2}$ Department of Earth and Environmental Sciences, Ludwig-Maximilians-Universität München, \\ 80333 München, Germany.
}

Received 2019 May 17; in original form 2019 May 17

\section{SUMMARY}

Local small-scale heterogeneities, like geological inhomogeneities, surface topographies and cavities are known to affect seismic wavefield gradients. This effect is in fact measurable with current instruments. For example, the agreement between data and synthetics computed in a tomographic model is often not as good for rotation as it is for displacement. Here, we use the theory of homogenization to explain why small-scale heterogeneities strongly affect wavefield gradients, but not the wavefield itself. We show that at any receiver measuring wavefield gradient, small-scale heterogeneities cause the wavefield gradient to couple with strain through a coupling tensor $\mathbf{J}$. Furthermore, we show that this $\mathbf{J}$ is (1) independent of source, (2) independent of time, but (3) only dependent on the receiver location. Consequently, we can invert for $\mathbf{J}$ based on an effective model for which synthetics fit displacement data reasonably well. Once inverted, $\mathbf{J}$ can be used to correct rotations at that receiver for other sources. Results of the correction are shown for synthetic rotational receivers and for the ring laser located in Wettzell, Germany. We find that compared to synthetics, the synthetics corrected through $\mathbf{J}$ are a better fit to the rotation data. Although results here are derived for rotations, they can be extended to receivers measuring any wavefield gradient. 
Key words: Site effects; Theoretical Seismology; Rotational Seismology.

\section{INTRODUCTION}

Seismic sources produce not only translations but also wavefield gradients, i.e. strains and rotations. Therefore, to assess any ground motion completely, the six components of strain and the three components of rotation are as important as the three components of translation (Aki \& Richards 2002). Collocated measurements of motions can be of great benefit for seismology because they allow us to retrieve instantaneous slowness without using travel times (Edme \& Yuan 2016; Wassermann et al. 2016; Bernauer et al. 2012; Fichtner \& Igel 2009; Igel et al. 2007, 2005; Mikumo \& Aki 1964), to constrain seismic source properties (Donner et al. 2017, 2016; Reinwald et al. 2016; Bernauer et al. 2014; Takeo \& Ito 1997) and to estimate parameters like propagation direction (Igel et al. 2007; Cochard et al. 2006) and rupture history (Takeo \& Ito 1997). Concurrent measurements of all three kinds of motions also help us remove the coupling that one motion introduces on the other, for example, horizontal rotations on translation (Aki \& Richards 2002, p. 604) and vertical rotation on translation (Graizer 2009; Trifunac \& Todorovska 2001). Further applications of collocated measurements have been discussed for example, in Schmelzbach et al. (2018), Li \& van der Baan (2017) and Igel et al. (2015).

Wavefield gradients themselves are important to deduce the stresses caused by seismic waves (Spudich et al. 1995), to enable S wave interpretation (Sollberger et al. 2016) and in earthquake engineering to assess seismic hazards (Basu et al. 2015; Castellani \& Boffi 1989; Abdel-Ghaffar \& Rubin 1984; Trifunac 1982).

Despite all these applications, measurements of wavefield gradients are relatively few. They were primarily estimated using dense arrays (Huang 2003; Bodin et al. 1997; Spudich et al. 1995) for a very long time because seismic instruments to measure them were either not reliable enough or sensitive enough in the required frequency bands, thus leading (Aki \& Richards 1980, p. 489) to note that seismology still awaits a suitable instrument for such measurements. Nowadays, rotations can be measured using liquid-based motion sensors (Egorov et al. 2015; Huang et al. 2013), magneto-hydrodynamic sensors (Pierson et al. 2016), ring laser gyroscopes (Schreiber et al. 2009; 
Pancha et al. 2000; McLeod et al. 1998), mechanical sensors (Brokešová \& Málek 2013) and more recently, using fiber-optic gyroscopes (Bernauer et al. 2018; Lindner et al. 2016; Kurzych et al. 2014). Similarly, strains can be measured using Distributed Acoustic Sensing (DAS) (Willis et al. 2016) and Distributed Vibration Sensing (DVS) (Dean et al. 2017).

Although considerable effort has been put into devising more robust and portable instruments, little attention has been paid to yet another issue concerning wavefield gradients. It has been shown that local small-scale heterogeneities, such as cavities and discontinuities, topographies as well as geological heterogeneities strongly affect strain (Gomberg \& Agnew 1996; Berger \& Beaumont 1976) and rotation (van Driel et al. 2012; Kohl \& Levine 1995; Harrison 1976; King \& Bilham 1973). This effect of heterogeneities on wavefield gradient is measurable with our current sensors. This leads to a number of consequences, such as 1) disagreement between wavefield gradient data and synthetics computed in a tomographic model, 2) dissimilarity between wavefield gradients measured at a point and wavefield gradients estimated at the point using an array, and 3) discrepancy between data observed among closely-spaced instruments. Such consequences may lead to an erroneous interpretation of the data.

The purpose of this paper is twofold. First, we study why wavefield gradients are affected by local small-scale heterogeneities. This will help us understand how a wavefield and its observables really interact with heterogeneities on all scales, which will eventually help us in better interpreting seismic data. Second, we investigate if it is possible to correct wavefield gradients for this effect. This will ensure a better agreement between wavefield gradient data and the synthetics that have been corrected. This will also help align the wavefield gradients measured at a point and wavefield gradients estimated at the point using an array.

The paper is organised as follows: we first describe an example in section 2 that will enable us to generalize the effect of small-scale heterogeneities on wavefield gradients. Using rotations and strains as our measurements, we show that indeed, small-scale heterogeneities strongly affect wavefield gradients. Then we approach the physics of this problem following the theory of homogenization. We demonstrate that there is a possibility of correcting for this effect. Finally, in section 3 , we show the results of this correction at a few synthetic rotational receivers before moving on 
to the G Ring laser in section 4. Although examples shown in 3 and 4 concern rotation, the results can be extended to any other wavefield gradient and their time derivatives.

\section{EFFECTS OF SMALL-SCALE HETEROGENEITIES ON WAVEFIELD GRADIENT MEASUREMENTS}

In this section, we begin with a simple numerical example where we show that the effect of smallscale heterogeneities is of a different nature on wavefield gradients, such as rotation and strain compared to the wavefield, such as displacement, velocity, and acceleration. This will be followed by theory in section 2.2 where we make use of homogenization to explain the observed effect and by a discussion in section 2.3 where we demonstrate the correction of this effect.

\subsection{Numerical Example}

We consider a 2D elastic case where the computational domains are two $20 \times 20 \mathrm{~km}^{2}$ squares as shown in Figure 1. These squares, except for spatially varying small-scale heterogeneity at the centre of the second (Fig. 1; model on the top-right panel), are otherwise homogeneous. The P and $\mathrm{S}$ velocities in the homogeneous area of the squares are $5 \mathrm{kms}^{-1}$ and $3.2 \mathrm{kms}^{-1}$ respectively. The spatial extension of the heterogeneity at the center of the second square is $200 \times 200 \mathrm{~m}^{2}$. Its geometry is kept simple in order to make both meshing and waveform modeling easier. The velocities in each element of the heterogeneity differ by $\pm 50 \%$ from the surroundings. This velocity contrast is strong but realistic for such a scale range. For example, the velocity contrast between granite and unconsolidated sediments is usually even larger. Finally, four receivers are placed in both squares such that for the heterogeneous square, two receivers lie just inside the heterogeneity and the other two just outside (Fig. 1; bottom panel). The distance between the receivers is around $15 \mathrm{~m}$. All boundaries are absorbing.

In both cases, we compute the wavefield induced by a horizontal double-couple source. The source-time function is a Ricker wavelet (second derivative of a Gaussian wavelet). With a source central frequency of $1.5 \mathrm{~Hz}$ (maximum frequency of $4.1 \mathrm{~Hz}$ ), the minimum wavelength $\lambda_{m}$ is around $700 \mathrm{~m}$. It should be noted that the dimension of the heterogeneity is around 3 times smaller 

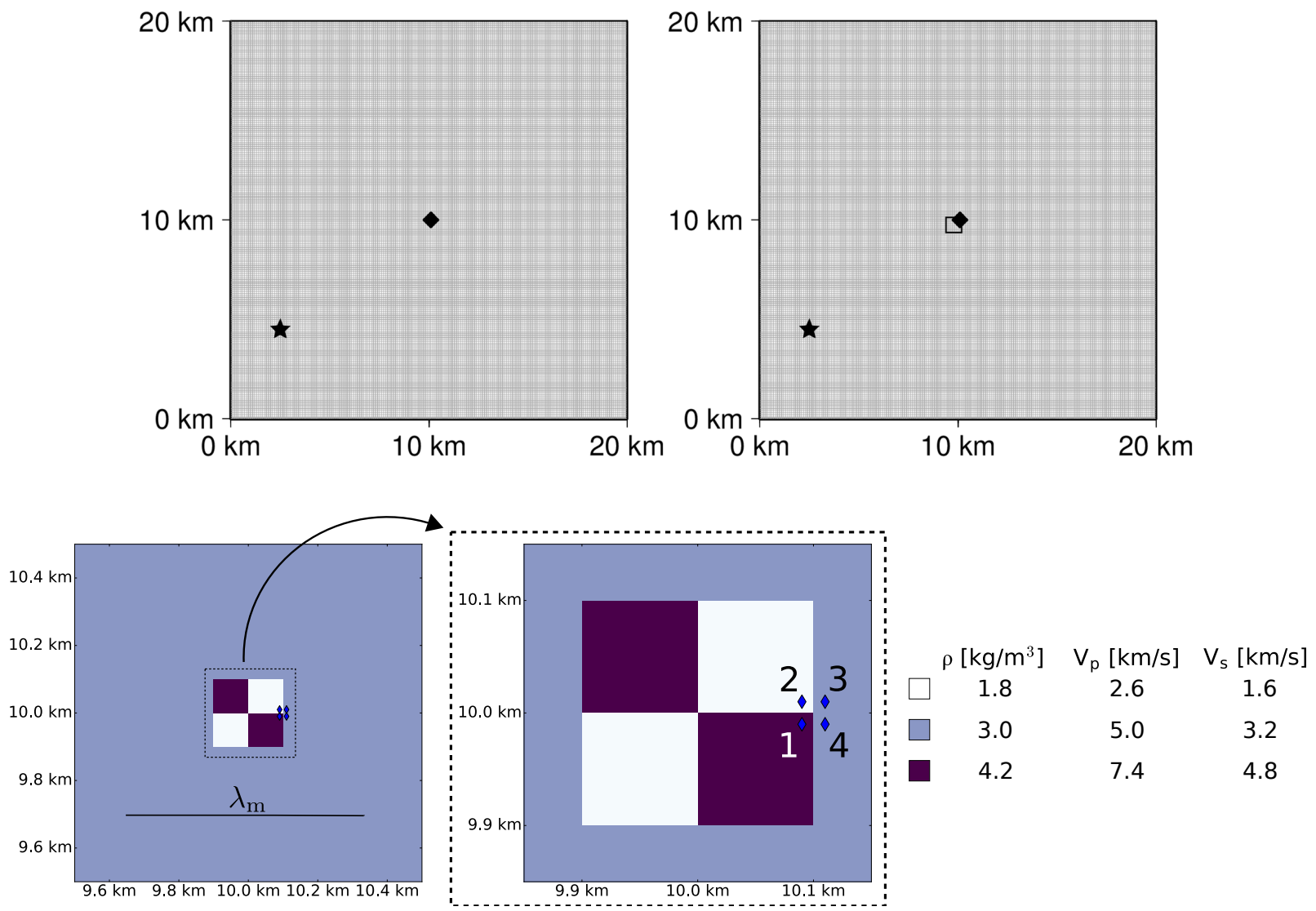

Figure 1. Top: 2D domains used in section 2.1. Both domains are $20 \times 20 \mathrm{~km}^{2}$. All boundaries are absorbing. The star shows the location of the source and the diamonds show the locations of four receivers. Left: Entirely homogeneous medium. Right: A heterogeneity of $200 \times 200 \mathrm{~m}^{2}$, placed at the centre, is shown by the square (not to scale).

Bottom: $\mathrm{V}_{\mathrm{p}}, \mathrm{V}_{\mathrm{s}}$ and $\rho$ at the heterogeneity. The length of the minimum wavelength for the simulation is shown in the leftmost panel.

than $\lambda_{m}$. Using a 2D Spectral Element (Komatitsch \& Vilotte 1998) program, displacements, velocities, accelerations, shear strain and rotation measured by the four receivers in the two models are computed. These are shown in Figure 2. Because the mesh used for the computation honors the interfaces of the heterogeneity, the solutions thus obtained are considered to be accurate.

It follows from Figure 2 that while all four receivers measure almost the same displacement, velocity and acceleration in both models, this is not true for rotation and strain. The four receivers measure a very similar rotation and strain in the homogeneous case, but in the presence of a heterogeneity, though much smaller than the minimum wavelength, the measurements are substantially different. The difference can be observed in both amplitudes and phases, and it depends on the rel- 


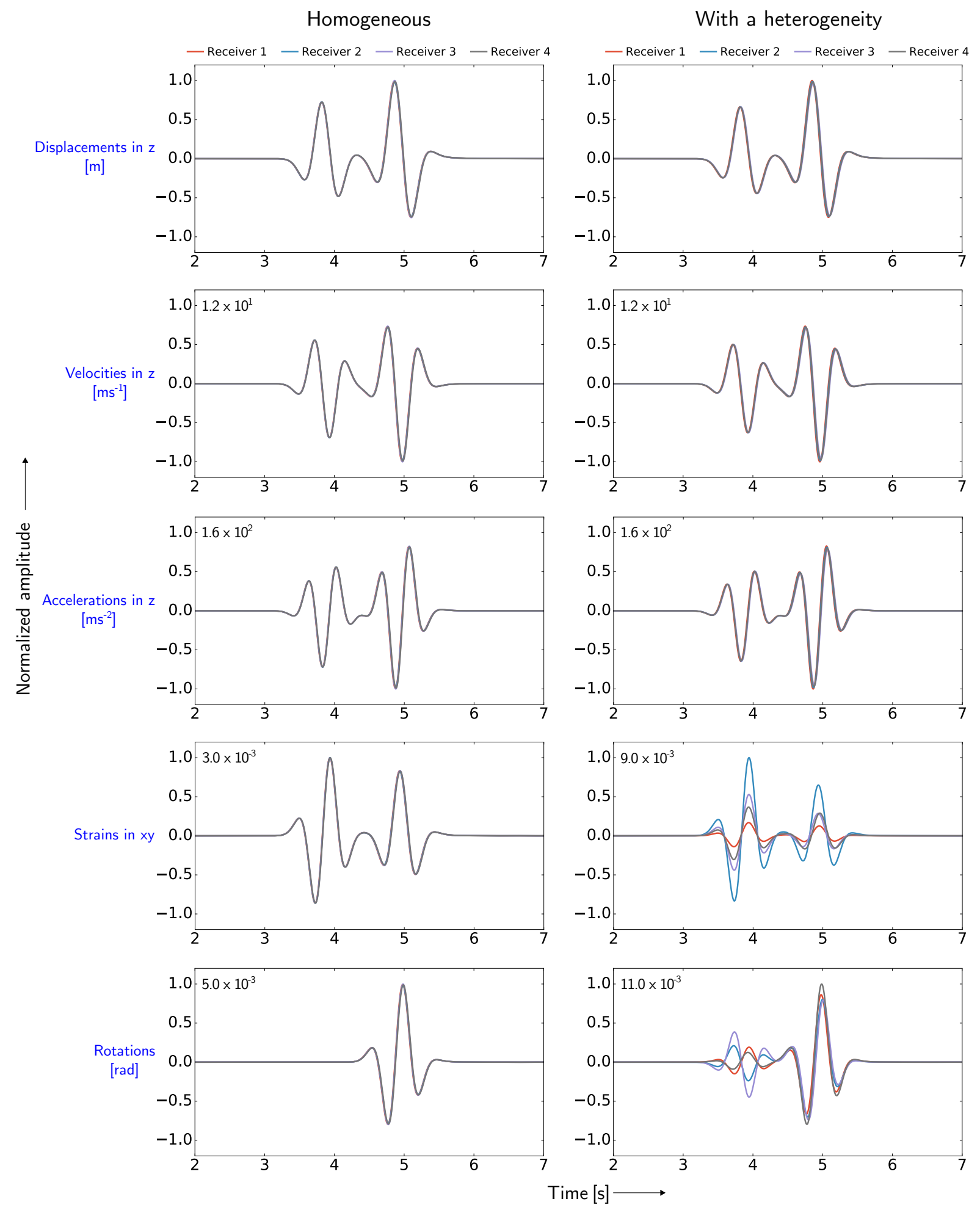

Figure 2. Left: Vertical displacements (top), vertical velocities (second), vertical accelerations (middle), shear strains (fourth) and rotations (bottom) in the homogeneous medium. Right: Vertical displacements (top), vertical velocities (second), vertical accelerations (middle), shear strains (fourth) and rotations (bottom) in the medium with heterogeneity. The scaling factors relative to the displacements for each panel are shown on the top left and the two media are shown in Figure 1. 
ative location of the receiver to the heterogeneity. Because our current sensors are sensitive enough to measure this difference, interpretations derived from the four receivers would be incoherent. For example, if these rotations were to be used to determine instantaneous slowness, one would arrive at four different values of slowness. Note that this result is not specific to just the quantities shown. The conclusion would be the same for any measurements of wavefield (and its time derivatives, such as displacement, velocity and acceleration) and for any measurements of wavefield gradient (and its time derivatives, such as strain, strain rate, rotation and rotation rate). It is also worth noting that acceleration is insensitive to the heterogeneity while rotation rate is. Because rotation rates are related to local phase velocities through transverse accelerations (Cochard et al. 2006; Igel et al. 2005; Pancha et al. 2000), this might seem inexplicable. However, the relation is only valid in the absence of heterogeneities, and as Figure 2 shows, it no longer holds when the receiver is placed at a heterogeneity. Similarly, Brokešová \& Málek (2015a) and Brokešová \& Málek (2015b) have shown that under certain conditions, velocities might be as important as accelerations for relating rotations to translations. However, as Figure 2 shows, the effect on rotations does not originate from velocities either.

From this simple numerical example, it can be concluded that small-scale heterogeneities have almost no effect on wavefield measurements but have a much stronger effect on wavefield gradient measurements. This observation is an issue for any wavefield gradient based instrument as the measurements can be tainted by nearby heterogeneities. A more intensive set of experiments would have shown that the amplitude of this effect depends mainly on the velocity contrast and not the heterogeneity's spatial extent with respect to the wavelength. In the following section, we examine this effect theoretically.

\subsection{Theory}

Homogenization deals with obtaining macroscopic, or homogenized or effective equations for systems with microscopic structures. In seismology, a microscopic medium is characterized by variations in elastic properties at scales much smaller than the minimum wavelength in the medium. By using homogenization, all these microscopic structures are smoothened out, i.e. the medium with 
rapidly-varying structures can be substituted with another where the structures are only slowlyvarying. To this end, homogenization has been beneficial in forward problem to study wave propagation in a fine-layered media (Backus 1962), to allow sparser meshing to gain computational speed (Cupillard \& Capdeville 2018; Capdeville et al. 2010a) as well as in the inverse problem to help interpret the results of full waveform inversion (Capdeville \& Métivier 2018). Because homogenization allows us to understand how different scales interact with the wavefield, it identifies itself as a natural choice to study the effects of small-scale structures on the gradients of displacement.

In the following, we give an intuitive construction of the homogenization problem. For simplicity, we consider a $2 \mathrm{D}$ periodic media where the properties repeat after certain intervals. For more extensive description on homogenization, Capdeville et al. (2010b,a); Cupillard \& Capdeville (2018) are recommended for 1D, 2D and 3D respectively.

We consider a wavefield $\mathbf{u}(\mathbf{x}, t)$ which is the solution of elastic wave equation for an external source $f(\mathbf{x}, t)$ with a maximum frequency $f_{\max }$. Because most geophysical media have no specific scale separation, we manually assign a separation scale $\lambda$. All spatial variations of the wavefield at scales below $\lambda$ are considered small-scales whereas all those above $\lambda$ are considered large-scales. Accordingly, we introduce the parameter $\varepsilon$,

$$
\varepsilon=\frac{\lambda}{\lambda_{m}}
$$

where $\lambda_{m}$ denotes the minimum spatial wavelength of the wavefield $\mathbf{u}$.

To separate large scales from small scales explicitly, we introduce a new space variable $\mathbf{y}$,

$$
\mathbf{y}=\frac{\mathbf{x}}{\varepsilon}
$$

$\mathbf{y}$ represents all the small-scales and $\mathbf{x}$ represents all the large-scales. It is then assumed that $\mathbf{x}$ and $\mathbf{y}$ can be treated as independent variables. This two-scale assumption can be mathematically justified by demonstrating its convergence towards the solution of a one-scale variable (for example, Dal Maso 1993; Allaire 1992). Consequently, all gradients with respect to $\mathrm{x}$ are redefined 
as

$$
\nabla_{\mathbf{x}} \rightarrow \nabla_{\mathbf{x}}+\frac{1}{\varepsilon} \nabla_{\mathbf{y}}
$$

where $\nabla_{\mathbf{x}}={ }^{t}\left(\partial_{x 1}, \partial_{x 2}\right)$ and $\nabla_{\mathbf{y}}={ }^{t}\left(\partial_{y 1}, \partial_{y 2}\right)$.

The aim of homogenization is to find solutions to the wave equations as an asymptotic expansion in $\varepsilon$ [see Appendix A]. As such, the equations for true displacement $\mathbf{u}^{\varepsilon}$ and true gradient $(\nabla \mathbf{u})^{\varepsilon}$ take the form

$$
\begin{aligned}
\mathbf{u}^{\varepsilon}(\mathbf{x}, \mathbf{y}, t) & =\mathbf{u}^{0}(\mathbf{x}, \mathbf{y}, t)+\varepsilon \mathbf{u}^{1}(\mathbf{x}, \mathbf{y}, t)+\varepsilon^{2} \mathbf{u}^{2}(\mathbf{x}, \mathbf{y}, t)+\ldots \\
(\nabla \mathbf{u})^{\varepsilon}(\mathbf{x}, \mathbf{y}, t) & =(\nabla \mathbf{u})^{0}(\mathbf{x}, \mathbf{y}, t)+\varepsilon(\nabla \mathbf{u})^{1}(\mathbf{x}, \mathbf{y}, t)+\varepsilon^{2}(\nabla \mathbf{u})^{2}(\mathbf{x}, \mathbf{y}, t)+\ldots
\end{aligned}
$$

In (4), the superscripts are powers in $\varepsilon$ but not in the $\mathbf{u}$ and $(\nabla \mathbf{u})$.

Solving the series of homogenization equations [see Appendix A], it can be shown that,

$$
\begin{aligned}
& \mathbf{u}^{0}(\mathbf{x}, \mathbf{y}, t)=\mathbf{u}^{0}(\mathbf{x}, t) \\
& \mathbf{u}^{1}(\mathbf{x}, \mathbf{y}, t)=\chi(\mathbf{y}): \boldsymbol{\epsilon}\left(\mathbf{u}^{0}(\mathbf{x}, t)\right)
\end{aligned}
$$

In (6), $\boldsymbol{\epsilon}$ denotes the strain $\left(\left[\epsilon\left(\mathbf{u}^{0}\right)\right]_{i j}=\frac{1}{2}\left(\partial_{i} u_{j}^{0}+\partial_{j} u_{i}^{0}\right)\right)$ and is not to be confused with the homogenization parameter $\varepsilon$ from (1). $\chi$, the first-order corrector, is a third-order tensor. It is a solution of the cell equations [see Appendix A]. Note that $\chi$ does not depend on time or sources.

Therefore, to leading order, the true displacement $\mathbf{u}^{\varepsilon}$ can be rewritten as

$$
\mathbf{u}^{\varepsilon}(\mathbf{x}, \mathbf{y}, t)=\mathbf{u}^{0}(\mathbf{x}, t)
$$

The true gradient $(\nabla \mathbf{u})^{\varepsilon}$ to the leading order is then

$$
(\nabla \mathbf{u})^{\varepsilon}(\mathbf{x}, \mathbf{y}, t)=\left(\nabla_{\mathbf{x}} \mathbf{u}^{0}\right)(\mathbf{x}, t)+\nabla_{\mathbf{y}} \chi(\mathbf{y}): \epsilon\left(\mathbf{u}^{0}(\mathbf{x}, t)\right)
$$

This implies that for rotation in $2 \mathrm{D}$, which is a scalar,

$$
\begin{aligned}
\omega^{\varepsilon} & =\theta_{i j}\left(\partial_{i} u_{j}\right)^{0} \\
& =\omega^{0}+J_{i j}: \epsilon_{i j}\left(u^{0}\right),
\end{aligned}
$$


where $J_{i j}=\theta_{m n} \partial_{y_{m}} \chi_{i j n}$ and $\theta$ represents the Levi-Civita symbol. Similarly, for rotation in 3D,

$$
\begin{aligned}
\omega_{i}^{\varepsilon} & =\theta_{i j k}\left(\partial_{j} u_{k}\right)^{0} \\
& =\omega_{i}^{0}+J_{i j k}: \epsilon_{j k}\left(u^{0}\right),
\end{aligned}
$$

where $J_{i j k}=\theta_{i m n} \partial_{y_{m}} \chi_{n j k}$ and $\theta$ represents the Levi-Civita symbol. And for a rotational receiver on the surface of a 3D domain under free surface conditions,

$$
\omega_{i}^{\varepsilon}=\omega_{i}^{0}+\bar{J}_{i j k}: \sigma_{j k}
$$

where $\bar{J}_{i j k}=J_{i m n} s_{m n j k}, \boldsymbol{\sigma}$ is the stress tensor and $\mathbf{s}$ is the inverse of the elastic tensor $\mathbf{c}$.

In (4), $\mathbf{u}^{\varepsilon}$ is the true displacement and $\mathbf{u}^{0}$ is the zeroth order effective displacement. Similarly, $(\nabla \mathbf{u})^{\varepsilon}$ represents the true gradient of displacement and $(\nabla \mathbf{u})^{0}$ represents the zeroth order effective gradient of displacement. True quantities correspond to real data that can be collected in the field. In theory, it is possible to model them using wave equations but because they depend on all possible scales, they require an infinite scale knowledge of the elastic properties of the Earth. This is not feasible in practice. On the other hand, for a given frequency band, effective quantities can be modeled using the same equations but using homogenized elastic properties which do not contain any small scales. Thus, effective quantities can be described with a finite set of parameters. Retrieving these parameters is what is actually done in full waveform inversion and tomography imaging (Capdeville \& Métivier 2018). Most of the tomographic models that exist are therefore actually an approximation to the homogenized Earth.

Comparing the terms in (7), we note that the terms on the right depends only on $\mathrm{x}$, i.e. largescales. This means that effective displacement can be used as good approximation for true displacement without worrying about small-scales. This is a well known result from Chapman (2004, p. 277) and Backus (1962). One consequence of this result is that the agreement between data and synthetics is reasonably good for displacement, velocity and acceleration. This is also consistent with the results obtained from section 2.1 and with many examples concerning real data. For instance, very long period surface waves can be modelled using PREM with good accuracy even though the crust is highly heterogeneous. The large amplitude and small-scale heterogeneity of 
the crust is inconspicuous to the displacement field, and one does not need complete information on the crust for its modeling.

On the contrary, this is not true for any gradient of displacement. The terms in (8) depend on both $\mathbf{x}$ and $\mathbf{y}$, i.e. both large-scales and small-scales. Due to small-scales, the true gradient of displacement now couples with strain of effective displacement. The coupling is due to the firstorder corrector $\chi$, therefore, we refer to this coupling as 'correction'. Similarly, we refer to $\mathbf{J}$ as the coupling tensor.

The appearance of correction in (8) implies that setting up an inversion problem with wavefield gradient is impossible because observations rely on all scales. Every detail in all scales would be necessary to set up the inversion problem. For the case of displacement, velocity or acceleration where effective quantities are a good approximation to the true quantities, this is not the case and setting up an inversion problem is not an issue. For the wavefield gradients, however, correction needs to be accounted for and this is the purpose of the next section.

\subsection{Inversion of the corrector}

We know from (9), (10) and (11) that the true wavefield gradient is coupled with strain of the effective displacement through the tensor $\mathbf{J}$. Moreover, $\mathbf{J}$ depends neither on time nor on source. It depends only on the location of the receiver. If the elastic properties at the receiver are known well enough, $\mathbf{J}$ can be computed by solving the cell problem (Capdeville et al. 2010a,b). This is not the case in practice. Nevertheless, we can invert for $\mathbf{J}$ provided that we have an effective model that fits the displacement (or velocity or acceleration) data reasonably well. The obvious choice for this effective model is the homogenized model because this is what the waves can see (Capdeville \& Métivier 2018). This effective model is usually not known, but any tomographic model can be a good approximation as we shall see from the synthetic examples in section 3. For real data, any Earth model which fits the displacement (or velocity or acceleration) data reasonably well can be used. This is similar to an earthquake moment tensor inversion where an Earth model is necessary to compute Green's functions. 
Because all examples in this paper are carried out for rotation, we start from the equation

$$
\omega^{\varepsilon}\left(\mathbf{x}_{r}, t ; \mathbf{x}_{s}\right)=\omega^{0}\left(\mathbf{x}_{r}, t ; \mathbf{x}_{s}\right)+\mathbf{J}\left(\mathbf{y}_{r}\right): \boldsymbol{\epsilon}^{0}\left(\mathbf{x}_{r}, t ; \mathbf{x}_{s}\right),
$$

where $\omega^{\varepsilon}$ is the rotation data, $\omega^{0}$ and $\epsilon^{0}$ are the rotation and the strain computed in the effective model and $\mathbf{J}$ is the unknown. $\mathbf{x}_{s}$ denotes the location of the source and $\mathbf{y}_{r}=\frac{\mathbf{x}_{r}}{\varepsilon}$ denotes the location of the receiver. We invert for $\mathbf{J}$ by solving the over-determined linear problem in the least-squares sense (Tarantola \& Valette 1982). We seek to minimise the $L^{2}$ misfit between the synthetics and the data through

$$
\mathbf{J} \sim\left({ }^{t} \mathbf{G} \mathbf{G}\right)^{-1} \mathbf{G} \delta d
$$

where $\delta d=\omega^{\varepsilon}-\omega^{0}$ and $\mathbf{G}$ is the matrix where all strains of the effective displacement are stacked.

The inversion process is straight-forward: we need only one simulation per source to assemble G. Then, we apply Equation (13) with no modification as the inversion is stable and no damping is necessary. We invert for 3 parameters in 2D and for 18 parameters for the 3 components of rotation in 3D. Like the instrument response, the corrector can be used to separate unwanted site effects from data.

Once we invert for $\mathbf{J}$, we need to evaluate the quality of our inversion results. One way to do so would be to compare the $\mathbf{J}$ computed through inversion to the one obtained by solving the series of homogenization equations [see the cell problem in Appendix A]. However, this solution is only available when all fine-scales are perfectly known, i.e only for synthetic tests. Therefore, for all our examples, we start by dividing the data into two pools: the inversion data set and the validation data set. We use the inversion data set to perform the inversion and the validation data set to assess the accuracy of $\mathbf{J}$. In all examples that follow, we use the $\mathbf{J}$ to correct the synthetic rotations. Therefore, a better fit between observed data and corrected synthetics, especially for events not used in the inversion, implies that $\mathbf{J}$ is a good measure of the coupling due to small-scales at the receiver. 


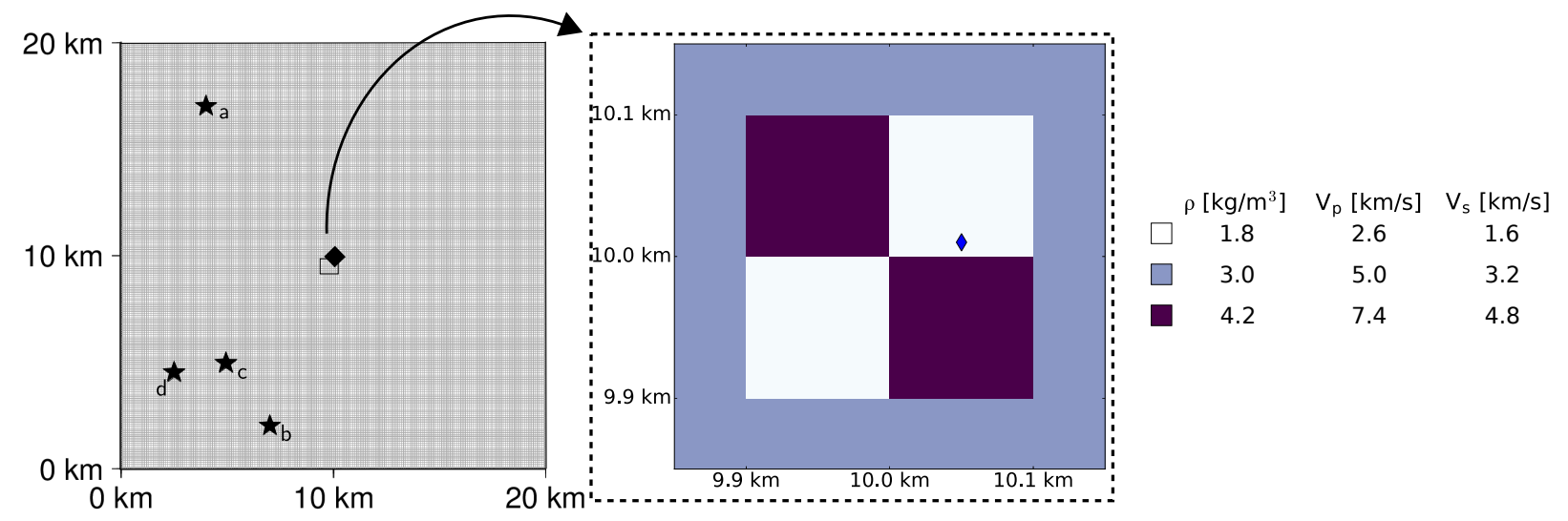

Figure 3. Left: 2D domain used in section 3.1. The domain is $20 \times 20 \mathrm{~km}^{2}$ and the heterogeneity at the centre, not to scale, is $200 \times 200 \mathrm{~m}^{2}$. The stars show the location of the sources and the diamond shows the location of the receiver. All boundaries are absorbing. Middle: Zoom in into the heterogeneity. Right: $\mathrm{V}_{\mathrm{p}}, \mathrm{V}_{\mathrm{s}}$ and $\rho$ in the domain. The $\mathrm{P}$ and $\mathrm{S}$ velocities in the outer domain are $5 \mathrm{kms}^{-1}$ and $3.2 \mathrm{kms}^{-1}$, and velocities for each element inside the heterogeneity are $\pm 50 \%$ of the surrounding domain.

\section{SYNTHETIC TESTS}

To test inversion of the coupling tensor $\mathbf{J}$, we first conduct synthetic tests. In section 3.1, we invert for $\mathbf{J}$ for a rotational receiver in 2D. This will be followed in section 3.2 where we invert for $\mathbf{J}$ for a rotational receiver placed on the free surface of a 3D domain. The set up that has been considered in both cases is quite simple in the sense that it does not take into account factors like attenuation and source uncertainties. All simulations are conducted using the Spectral Element Method (SEM) that solves the elastic wave equation.

In both cases, the sources are either forces or moment tensors but we always choose the Ricker wavelet as the source-time function.

\subsection{Inversion of $\mathbf{J}$ for a receiver in a $2 \mathrm{D}$ volume}

Our first model is a 2D domain whose $\mathrm{P}$ and $\mathrm{S}$ velocities are $5 \mathrm{kms}^{-1}$ and $3.2 \mathrm{kms}^{-1}$ respectively. The size of the domain is $20 \times 20 \mathrm{~km}^{2}$. A heterogeneity of size $200 \times 200 \mathrm{~m}^{2}$ is at the center of the domain and the $\mathrm{P}$ and $\mathrm{S}$ velocities in each element in the heterogeneity are $\pm 50 \%$ of the surrounding domain. The geometric configuration of the model is shown in Figure 3.

Because the rotational receiver has been placed in the heterogeneity, we expect that the heterogeneity affects the rotation measurements. We therefore invert for the coupling tensor $\mathbf{J}$. To 
this end, we first perform three simulations in this medium with sources at positions shown by the stars $\mathrm{a}, \mathrm{b}$ and $\mathrm{c}$ respectively in Figure 3 to generate the data (Fig. 4; black lines). These rotations make up the term on the left in Equation 12. The central frequency of all three sources is $1.5 \mathrm{~Hz}$ (maximum frequency $4.1 \mathrm{~Hz}$ ). Two of the sources considered are double-couple, while one is a force. Although the heterogeneity is much smaller than $\lambda_{m}$, the spectral element mesh for the simulation honors all interfaces of the heterogeneity. The rotations computed are therefore considered accurate.

Next, we need to choose an effective model for the inversion. Certainly, we could use the homogenized version of the original medium but with an underlying assumption that the heterogeneous area has negligible effect on the effective model, we use a homogeneous model instead. This assumption is valid because the displacements in both media are in good agreement [see Appendix C]. Moreover, it is close to realistic situations where tomographic models are far from perfect.

We use the same three sources as before in this homogeneous model and compute the rotations and strains at the receiver. These are the two known quantities on the right in Equation 12. The rotations measured in the homogeneous model are shown in Figure 4 (green lines). Comparison of the rotations measured in the heterogeneous and homogeneous case in the figure reveals a significant difference in all four panels. Clearly, the difference is due to the presence of the heterogeneity and it depends on the path that the waveform takes through the heterogeneity.

We use the data and synthetics thus obtained to invert for $\mathbf{J}$. The fact that we use SEM to both generate data and to invert for $\mathbf{J}$ is not an inversion crime here. Indeed, it would be an inversion crime if the data were generated using the theory of homogenization and the inversion performed with the same mesh. Here, we generate the data using a fine mesh with an element size of $100 \mathrm{~m}$, i.e. without the theory of homogenization, but carry out the inversion on a sparse mesh with an element size of $200 \mathrm{~m}$.

Once we obtain $\mathbf{J}$, we use it to correct the synthetics, i.e. we add the last term in Equation 12 to the rotations measured in homogeneous medium. These are shown in Figure 4 (red lines). It can be seen that the corrected synthetic rotations are a much better fit to the heterogeneous rotations, but this is unsurprising because these data have been used for the inversion. Therefore, to truly render 

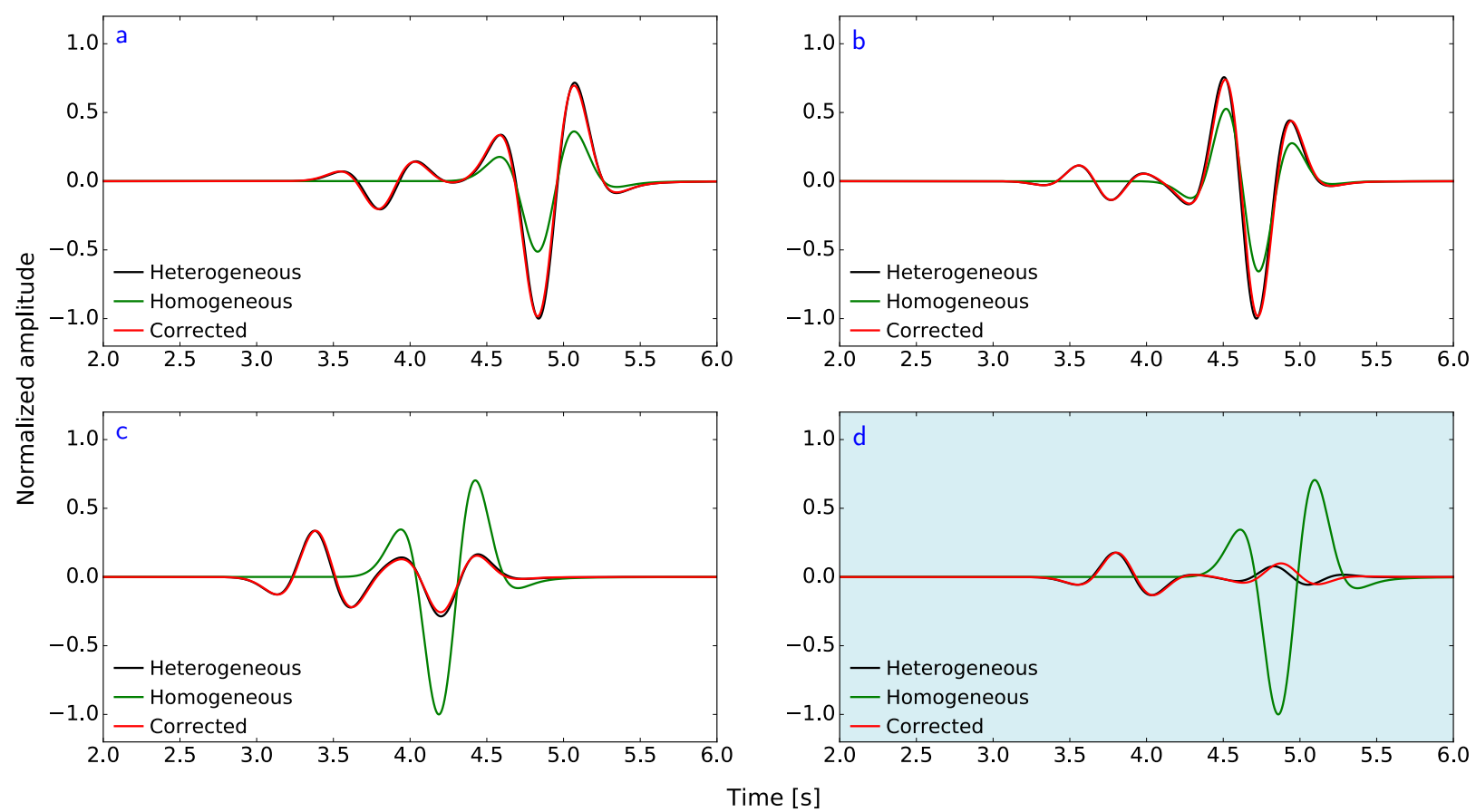

Figure 4. Rotations. Panels a, b, c: Three events used for inversion of the corrector. Panel d: The event is used as validation using this corrector. It is shown in blue to indicate that it has not been used for the inversion.

Heterogeneous and homogeneous rotations are shown in black and green respectively. Rotations that have been corrected using the coupling tensor $\mathbf{J}$ are shown in red.

its accuracy, we use this $\mathbf{J}$ to a new set of data which has not been used for the inversion (Fig. 4; Panel d) as validation; the new source positioned at the location shown by the star $\mathrm{d}$ in Figure 3. The new source is a vertical force. As expected, it can be seen that $\mathbf{J}$ is able to recover the rotation in the heterogeneous medium.

\subsection{Inversion of $\mathbf{J}$ for a receiver on a 3D surface}

Our second model is a 3D domain whose $\mathrm{P}$ and $\mathrm{S}$ velocities are $5 \mathrm{kms}^{-1}$ and $3.2 \mathrm{kms}^{-1}$ respectively. The domain size is $40 \times 40 \times 10 \mathrm{~km}^{3}$. A heterogeneity is placed at the center of the surface, with $\mathrm{P}$ and $\mathrm{S}$ velocities for each element in the heterogeneity chosen randomly and independently but within $25 \%$ of the surrounding domain. The spatial extent of the heterogeneity is $1334 \times 1334 \times 222 \mathrm{~m}^{3}$. The geometric configuration of the model is shown in Figure 5 .

Because the rotational receiver here is surrounded by the heterogeneity, the rotation measurements are most likely affected by the heterogeneity. We therefore seek for the coupling tensor $\mathbf{J}$ for 

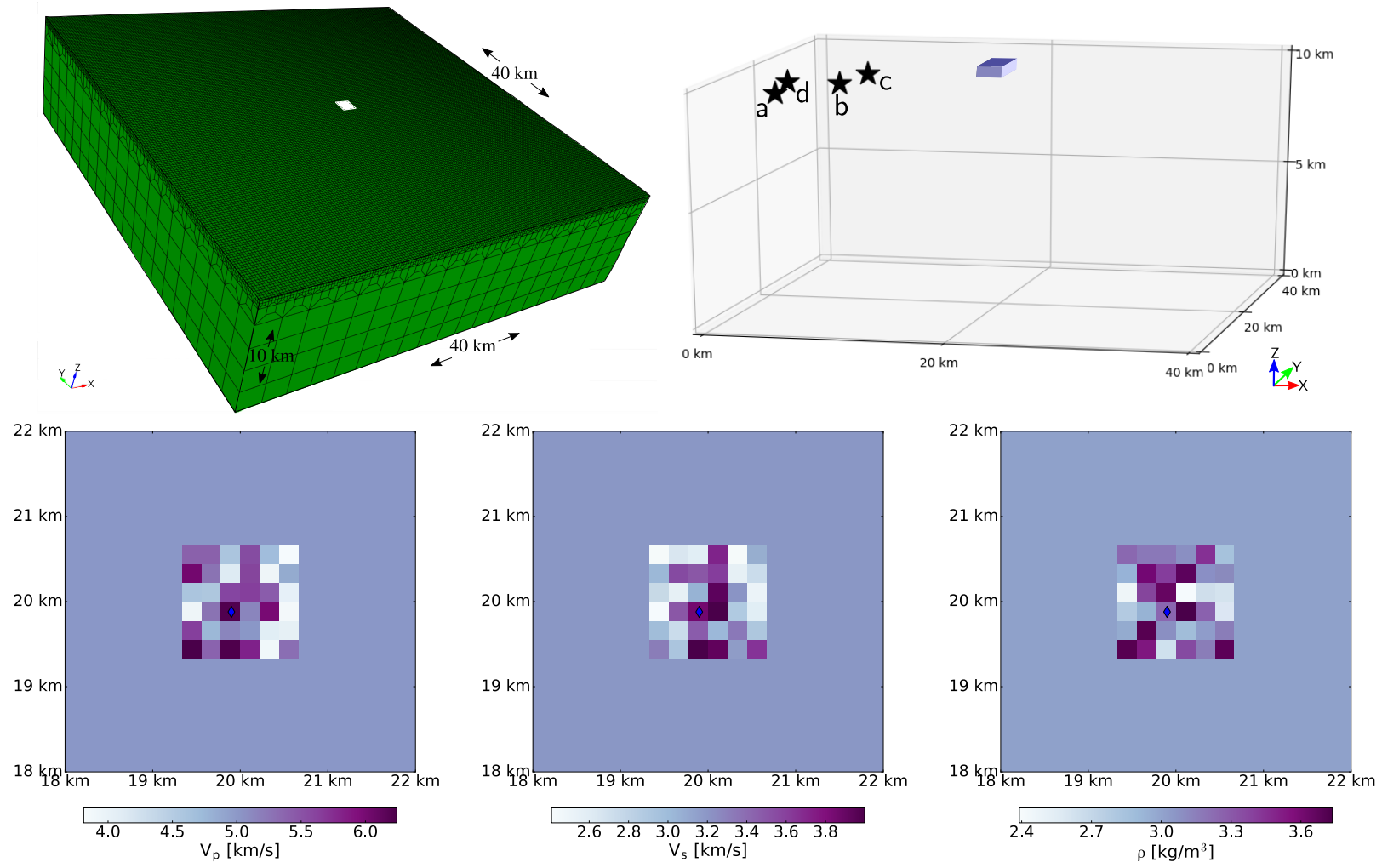

Figure 5. Top: 3D domain used in section 3.2. The domain is $40 \times 40 \times 10 \mathrm{~km}^{3}$, and the heterogeneity at the centre, not to scale, is $1334 \times 1334 \times 222 \mathrm{~m}^{3}$. The stars show the location of the sources and the diamond shows the location of the receiver. All but top boundary are absorbing.

Bottom: Zoom in into the heterogeneity from the top showing the $\mathrm{V}_{\mathrm{p}}, \mathrm{V}_{\mathrm{s}}$ and $\rho$ in the domain. The $\mathrm{P}$ and $\mathrm{S}$ velocities in the outer domain are $5 \mathrm{kms}^{-1}$ and $3.2 \mathrm{kms}^{-1}$, and velocities for each element inside the heterogeneity are chosen randomly and independently, but within $25 \%$ of the surrounding domain.

this receiver. Here, we only invert for $\mathbf{J}$ for one component of the rotation, i.e. rotation around the z-axis. This allows us to invert for just 6 parameters instead of 18. Note that in this case - where the receiver has been placed at the surface - the free surface condition also allows us to further reduce the number of scalars to be inverted for to 3 (Equation 11). To this end, we perform simulations for three different sources at locations shown by the stars a, b and c in Figure 5 in the original medium to generate data (Fig. 6; black lines). These rotations form the term on the left in Equation 12. All sources have a central frequency of $0.5 \mathrm{~Hz}$ (maximum frequency $1.4 \mathrm{~Hz}$ ). Two of the sources are forces, while the third one is a moment tensor. Once again, the mesh honors all boundaries and therefore, the solutions obtained are considered accurate.

Similar to section 3.1, we carry out the inversion using the homogeneous medium as an ap- 
proximation for the effective one. We use the same three sources in the homogeneous medium to generate rotations and strains. These are the two known quantities on the right in Equation 12. The rotations in this tomographic medium are shown in Figure 6 (green lines). Comparing the rotations in the heterogeneous and the homogeneous media, we see that the waveform is altered in all four cases owing to the heterogeneity.

We use the data and synthetics thus derived from these simulations to invert for the coupling tensor $\mathbf{J}$ which is in turn used to correct the synthetic rotations (Fig. 6; red lines). The corrected synthetic rotations are a much better fit to the heterogeneous rotations, which is expected because these data have been used for the inversion. Finally, we perform one last simulation with the source, which is a moment tensor, at location shown by the star $\mathrm{d}$ in Figure 5 to validate the coupling on a new set of data (Fig. 6; Panel d), one that has not been used previously for the inversion. Doing this allows us to estimate the accuracy of $\mathbf{J}$. Unsurprisingly, the recovery of the heterogeneous rotation is achievable through $\mathbf{J}$.

It is important to note that the correction is not always perfect, i. e. the corrected rotations do not always overlap the heterogeneous rotations. This is especially evident in panels a and d in Figure 6. The reasons for this are 1) the homogeneous model used here is not a perfect approximation to the homogenized or effective model and 2) the method, being asymptotic, implies some errors.

\section{REAL DATA}

Ever since it's operation in 2001, the G Ring laser in Wettzell, $150 \mathrm{~km}$ north-east of Munich (Fig. 8; the seismometer WET and the G Ring laser are collocated), has been successfully recording the vertical component of rotation rates that are generated by seismic sources (Suryanto et al. 2006; Igel et al. 2005, 2007). Using two counter propagating beams of wavelength $633 \mathrm{~nm}$ to generate a beat frequency, it measures rotation rates as small as $10^{-10} \mathrm{rads}^{-1} \mathrm{~Hz}^{-1 / 2}$ (Schreiber et al. 2003).

Here, we invert for the coupling tensor $\mathbf{J}$ at this ring laser for long period teleseismic events. In section 4.1, we use the normal mode summation program to compute velocities and rotation rates in PREM (Dziewoński \& Anderson 1981). We show that the agreement between the rotation rates measured at the ring laser and the rotation rates computed in PREM is less compared to the one 

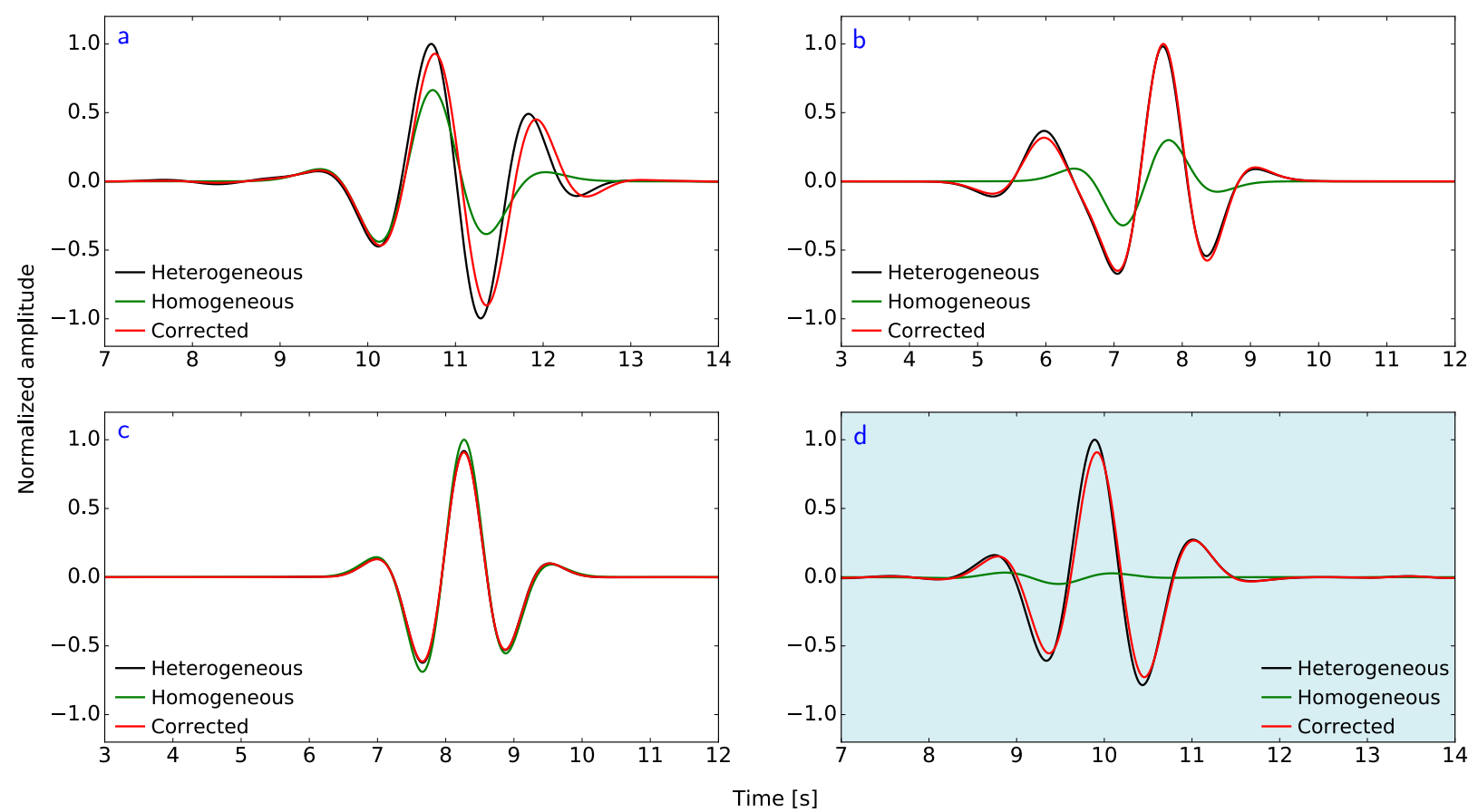

Figure 6. Rotations. Panels a, b, c: Three events are used for inversion of the corrector. Panel d: The event is used as validation using this corrector. It is shown in blue to indicate that it has not been used for the inversion.

Heterogeneous and homogeneous rotations are shown in black and green respectively. Rotations that have been corrected using the coupling tensor $\mathbf{J}$ are shown in red.

between velocities. We invert for the coupling tensor $\mathbf{J}$ and correct the rotation rates computed in PREM to improve the agreement. In section 4.2, we show that due to small-scale heterogeneities at the receiver, observations of rotation rates and array-derived estimations of rotation rates at the ring laser are not the same. We invert for the coupling tensor $\mathbf{J}$ and correct the array-derived rotation rates such that they are in better agreement to the observed rotation rates.

We use the definition of L2 norm defined below to quantify the misfit between the observed and synthetic rotation rates.

$$
\xi:=\frac{\sqrt{\int_{0}^{t_{\max }}\left(\dot{\omega}-\dot{\omega}^{d a t a}\right)^{2}(t) d t}}{\sqrt{\left(\dot{\omega}^{\text {data }}\right)^{2}(t) d t}} ;
$$

where $\dot{\omega}$ is the synthetic vertical rotation rate and $\dot{\omega}^{\text {data }}$ is the observed vertical rotation rate. Note that this misfit takes into account all parts of the seismogram. 


\subsection{Inversion for $\mathbf{J}$ at the Ring laser, Wettzell using PREM}

We use Obspy (Krischer et al. 2015) to get rotation rates recorded by the ring laser and velocities recorded by the collocated seismometer WET for all events between January 2007 and June 2018 and within $\mathrm{M}_{w} 7.0$ and 9.0. After download, all data are corrected for instrument response. Next, we compute synthetic velocities and rotation rates by using a normal mode summation program to solve the wave equation in a PREM with ocean and anisotropy. For this, centroid-moment tensor solutions from the GCMT catalog (Ekström et al. 2012) are used with a step function as the sourcetime function.

We compare the observed and synthetic velocities in order to remove observed data with no visible earthquake signal. Apart from that, no prior selection of events is done. A figure showing the source-receiver distribution in shown in Appendix D. Comparing the fit between observed and synthetic velocities to the fit between observed and synthetic rotation rates for all events within frequencies $0.002-0.009 \mathrm{~Hz}$, we find that the rotation rates are less in agreement compared to the velocities [see Appendix C]. Often, the misfits as defined in (14) are about 0.3 between the velocities but are greater than 0.7 between the rotation rates. We suspect this to be due to small-scale heterogeneities at the ring laser and therefore, invert for $\mathbf{J}$ using 32 events. The $\mathbf{J}$ thus obtained is in turn used to correct the synthetic rotation rates. One of such events is shown in Figure 7 (top panel). A validation data set is then set up to help access the quality of our results. It includes 32 events not used in the inversion. One of the events from this validation data set is shown in Figure 7 (bottom panel). For both panels, we see distinct arrivals of direct Love waves and indirect Love waves. We also see that the corrected rotation rates (red lines) are a better fit to the observed rotation rates (black lines) than the synthetic rotation rates (green lines). Misfits for about $72 \%$ of the events in the validation data set improve after the introduction of $\mathbf{J}$, proving that indeed, $\mathbf{J}$ is a good indication of the coupling. For example, the misfit for the event shown in the bottom panel of Figure 7 reduced from 0.86 to 0.63 . 


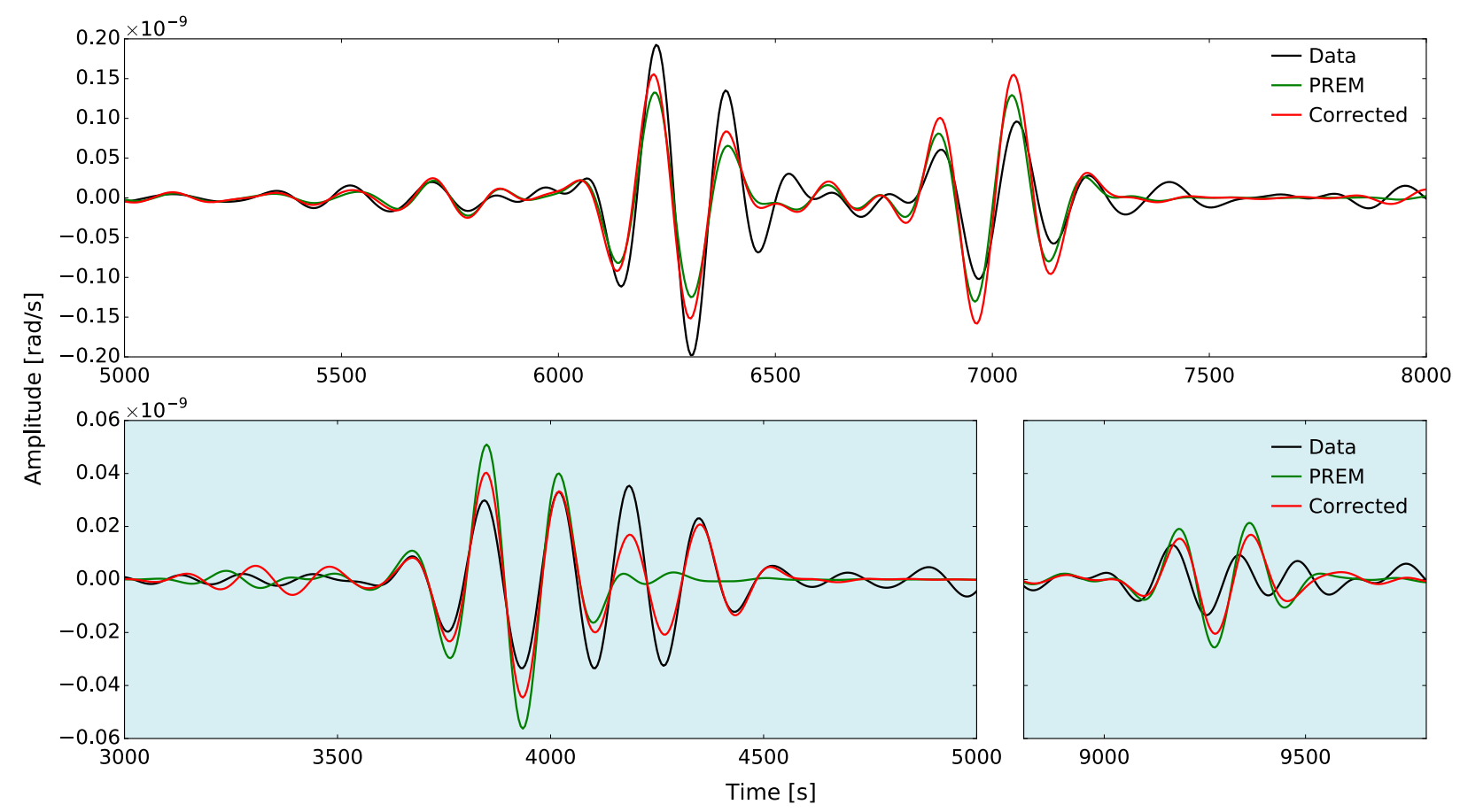

Figure 7. Rotation rates. Top: 201611131102A South Island, New Zealand. One of the 32 events that are used to invert for the coupling tensor J. Bottom: 201210280304A Queen Charlotte Islands. An event from the validation data set. It is shown in blue to indicate that this event has not been used for the inversion.

Data, synthetic and corrected rotation rates are given in black, green and red respectively.

\subsection{Inversion for $\mathbf{J}$ at the Ring laser, Wettzell using array-derived estimates}

In the absence of small-scale heterogeneities, wavefield gradients estimated at a point using the finite-difference of array data are the same as wavefield gradients measured at the point. In practice, this is not the case. Wavefield gradients estimated from an array have their coupling tensor although it appears in a slightly different form [see Appendix B] than that of wavefield gradients measured at a point. In the case of rotation, the two coupling tensors are related through the following equations:

$$
\begin{gathered}
\omega(\mathbf{x}, \mathbf{y}, t)=\omega^{0}(\mathbf{x})+\mathbf{J}(\mathbf{y}): \boldsymbol{\epsilon}\left(\mathbf{u}^{0}(\mathbf{x}, t)\right), \\
\omega^{\mathrm{AD}}(\mathbf{x}, \mathbf{y}, t)=\omega^{0}(\mathbf{x})+\mathbf{J}^{\mathrm{AD}}(\mathbf{y}): \boldsymbol{\epsilon}\left(\mathbf{u}^{0}(\mathbf{x}, t)\right),
\end{gathered}
$$

where $\omega^{0}$ is the rotation in an effective model. $\omega$ is the rotation measured at a point and $\mathbf{J}$ is its coupling tensor. Similarly, $\omega^{\mathrm{AD}}$ is the rotation estimated at a point using array derived measurements 

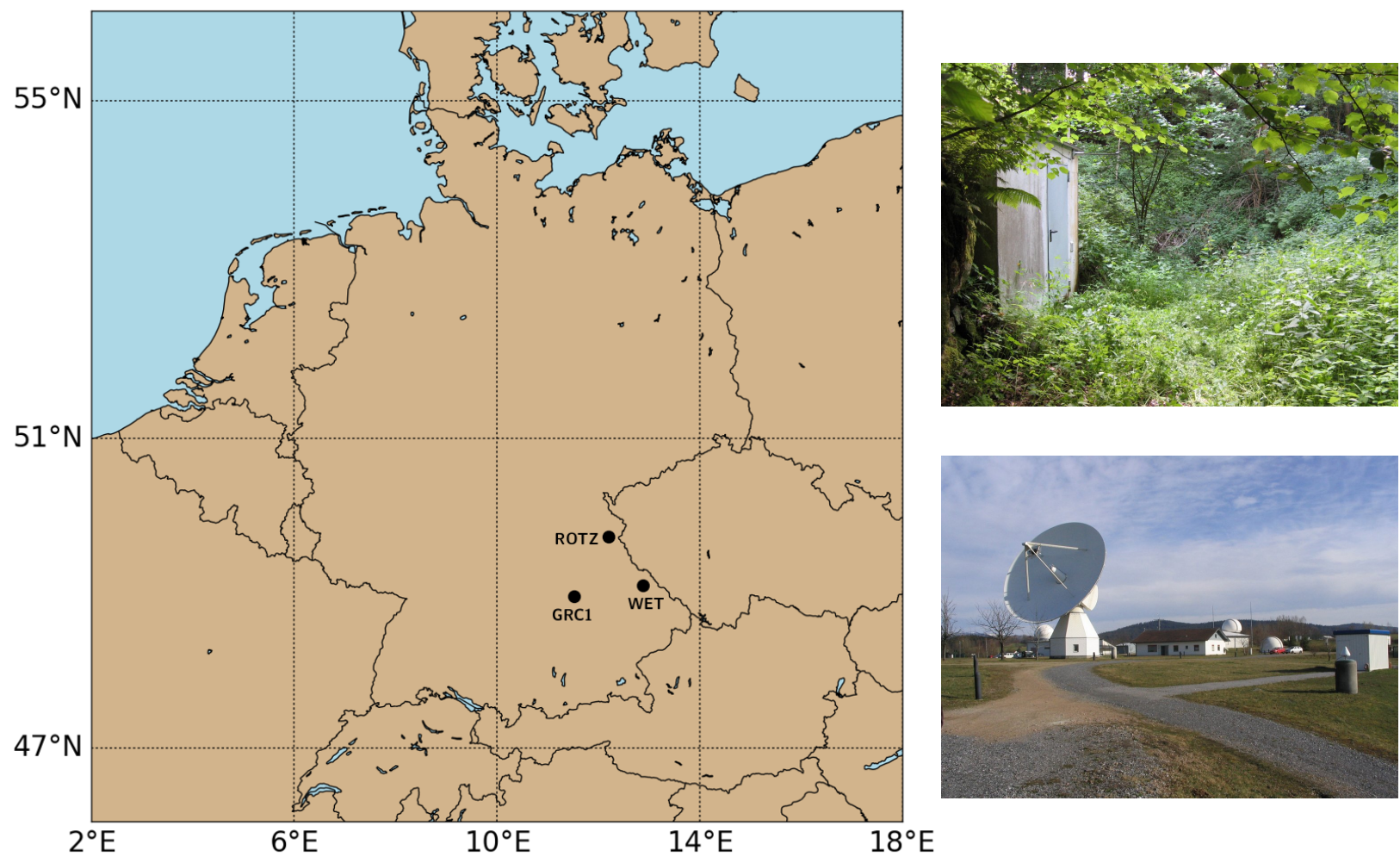

Figure 8. Left: location of the receivers used for the finite difference. Right: Location of the seismometer ROTZ (top) and WET (bottom).

and $\mathbf{J}^{\mathrm{AD}}$ is its coupling tensor. These equations lead to

$$
\omega(\mathbf{x}, \mathbf{y}, t)=\omega^{\mathrm{AD}}(\mathbf{x}, \mathbf{y}, t)+\mathbf{J}^{\dagger}(\mathbf{y}): \boldsymbol{\epsilon}\left(\mathbf{u}^{0}(\mathbf{x}, t)\right)
$$

where $\mathbf{J}^{\dagger}(\mathbf{y})=\mathbf{J}(\mathbf{y})-\mathbf{J}^{\mathrm{AD}}(\mathbf{y})$.

For our purpose, we use velocities recorded at stations WET, ROTZ and GRC1 (Fig. 8) to estimate array-derived rotation rates at the ring laser. Each station is a broadband STS-2 seismometer that measures velocity with a sampling rate of $20 \mathrm{~Hz}$. The distance between each station is in the order of $100 \mathrm{~km}$. Assuming an average phase velocity of $3 \mathrm{kms}^{-1}$, we look at all events between January 2007 and June 2018 for frequencies between 0.0005 and $0.003 \mathrm{~Hz}$ following Poppeliers \& Evans (2015) and Langston (2007).

We select only 7 events due to their good signal to noise ratio. A figure showing the sourcereceiver distribution in shown in Appendix D. Comparing the array-derived rotation rates to those observed at the ring laser, we find that the misfits are always above 0.75 even though the velocities from three seismometers are always in agreement [see Appendix C]. Therefore, we follow the 


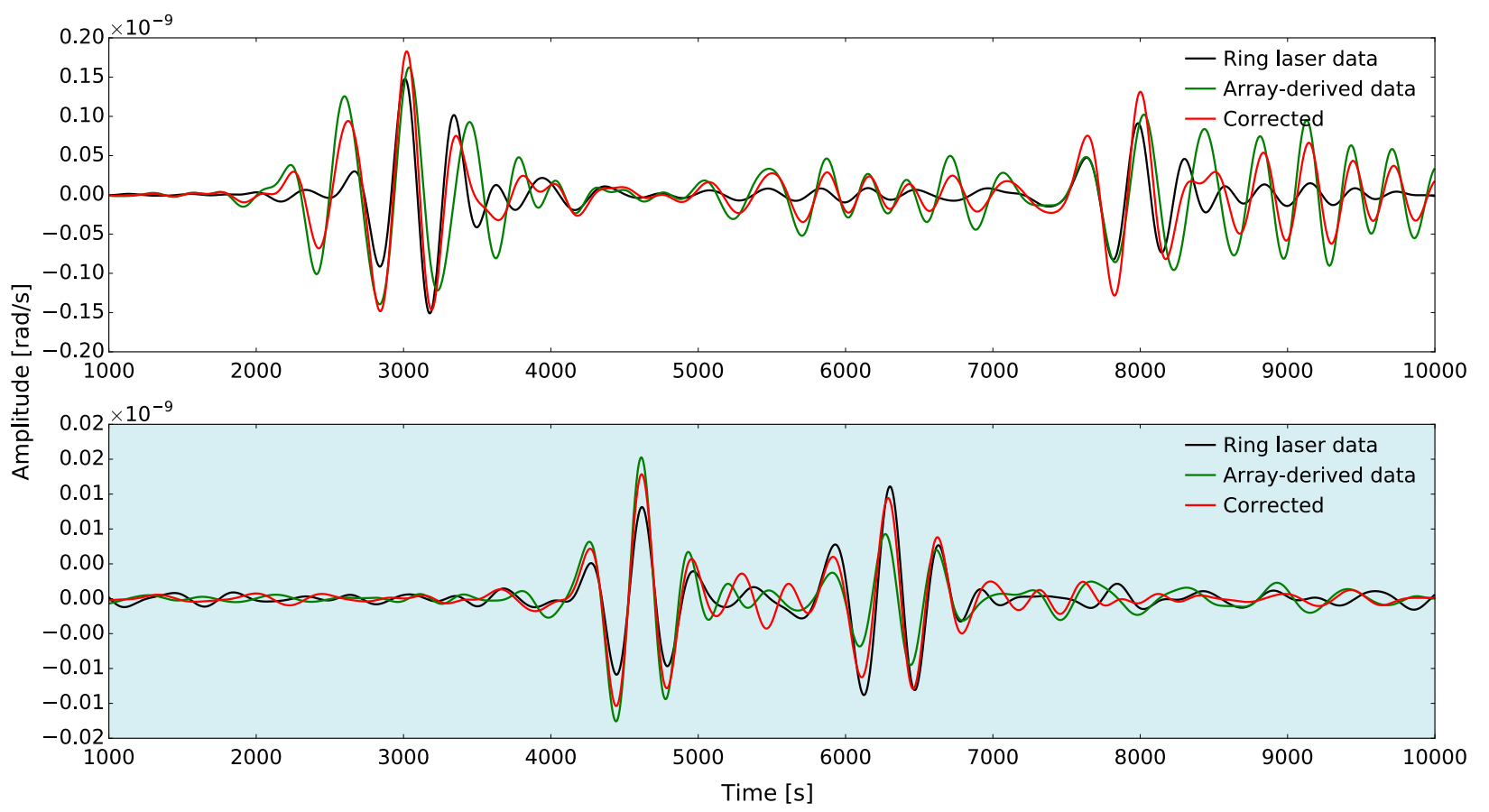

Figure 9. Rotation rates. Top: 201103110546A Near East Coast of Honsh. One of the 6 events that are used to invert for the coupling tensor J. Bottom: 200909291748A Samoa Islands Region. An event from the validation data set. It is shown in blue to indicate that this event has not been used for the inversion.

Observed, array-derived and corrected rotation rates are given in black, green and red respectively.

same procedure as in section 4.1 and use 6 events for the inversion of $\mathbf{J}^{\dagger}$. We then use $\mathbf{J}^{\dagger}$ to correct the array-derived rotation rates. One of these events is shown in Figure 9 (top panel). The validation data set in this case consists of 1 event that has not been used for the inversion. This event is shown in Figure 9 (bottom panel). For both events, we see distinct arrivals of direct Love waves and indirect Love waves phases. Furthermore, we see that the corrected rotation rates (red lines) are in better agreement to the observed rotation rates (black lines) than the array-derived rotation rates (green lines). In this case, the misfit for the validation data set was 0.66 originally. After the introduction of the coupling tensor however, the misfit improved to 0.45 .

Note that usually, when estimating array-derived rotations, the effect of small-scale structures is often ignored. In that case, many stations are needed to average out the effect and minimize the coupling tensor $\mathbf{J}$. Here, we explicitly account for the effect and therefore, it is futile to increase the number of stations. 


\section{DISCUSSION AND CONCLUSIONS}

In this study, through a simple example, we showed that local small-scale heterogeneities have little or almost no effect on the wavefield (displacement, velocity and acceleration) but a strong effect on its gradients (strain, strain rate, rotation and rotation rate). This stands in line with previous studies conducted by Gomberg \& Agnew (1996); Kohl \& Levine (1995); Lennon \& Baker (1973). Local small-scale heterogeneities can cause both phase and amplitude of the waveform to alter, thus causing a disagreement between the observed data and synthetics. Consequently, these effects are obstacles in direct applications of the measurements, for example, when rotations are used to determine phase velocities (Igel et al. 2005) or strains are used to deduce stresses generated by earthquakes (Spudich et al. 1995). Similarly, the free surface condition may not be valid (Gomberg \& Agnew 1996).

Through the theory of homogenization, we introduced the coupling tensor $\mathbf{J}$ and showed that at any receiver measuring wavefield gradient, small-scale heterogeneities cause the wavefield gradient to couple with the strain of effective displacement through $\mathbf{J}$. Therefore, a knowledge of $\mathbf{J}$ is needed to correct for this coupling. One option to determine $\mathbf{J}$ would be through direct computation, but this is only feasible if all fine-scales at the receiver are well-known. The second option is to determine $\mathbf{J}$ through linear inversion and it is indeed possible to perform this inversion because $\mathbf{J}$ does not depend on source or time. It is a characteristic of the receiver location. One necessary condition for this inversion however is that an effective model that fits displacement data well is needed. This is not an issue for most synthetic cases and for low frequency data where PREM can be used as the effective model.

We then inverted for $\mathbf{J}$ for rotational receivers in two synthetic cases, one in $2 \mathrm{D}$ and one in $3 \mathrm{D}$. In both these examples, we used the $\mathbf{J}$ to correct synthetic rotations and saw that the corrected synthetic rotations were a better match to the synthetic data as compared to synthetic rotations. This was also true for rotations that were not used for inversion. We conclude that although the effect of small-scale heterogeneities can be substantially large, it can be corrected for. The setting for our synthetic examples was one where the sources are either forces or moment tensors; however, further tests in different settings reveal the same conclusion. 
Next, we introduced $\mathbf{J}$ to rotation rates measured by the G Ring laser in Germany. It is important to note that data are unavoidably affected by factors such as topography, instrument errors, background noise and so on. Furthermore, the bedrock at Wettzell consists mostly of igneous and metamorphic rocks, thus suggesting a very small correction. Nevertheless, we saw that the misfit was better between the observed and the corrected rotation rates as compared to the one between observed and synthetic rotation rates.

One other consequence of small-scale heterogeneities is that wavefield gradients measured at a point do not coincide with wavefield gradients estimated at the point using an array. However, we showed that the coupling tensor $\mathbf{J}$ for one can be related to coupling tensor $\mathbf{J}$ of another. This was confirmed in the last section where we see a disagreement between the rotation rates estimated at the G Ring laser using an array and the rotation rates measured by it. We corrected for the arrayderived rotation rates and the agreement between corrected rotation rates and the rotation rates measured by the ring laser improved significantly.

Although all examples here are based on rotation and rotation rates, the results can be extended to any other wavefield gradient with no modifications. Future work will focus on the relationship between $\mathbf{J}$ and subsurface geology. This relationship is highly non-linear and strong apriori information with Bayesian style inversion will be needed.

\section{ACKNOWLEDGEMENTS}

We would like to thank Michael Afanasiev and Johana Brokešová whose comments have really helped improve the paper.

This work was funded by the French National Research Agency (ANR-16-CE31-0022). We also thank the support through the European Research Council (Advanced Grant: ROMY, number: 339991).

Numerical simulations were performed at CCIPL and we thank the people who work endlessly to maintain the system. Obspy was acquired from https://github.com/obspy/obspy/wiki and all event data were acquired from the global CMT catalog (Ekström et al. 2012). 


\section{REFERENCES}

Abdel-Ghaffar, A. M. \& Rubin, L. I., 1984. Torsional earthquake response of suspension bridges, Journal of Engineering Mechanics, 110(10), 1467-1484.

Aki, K. \& Richards, P., 1980. Quantitative Seismology, W. H. Freeman and Company.

Aki, K. \& Richards, P., 2002. Quantitative Seismology, 2nd Ed., University Science Books.

Allaire, G., 1992. Homogenization and two-scale convergence, SIAM Journal on Mathematical Analysis, 23(6), 1482-1518.

Backus, G. E., 1962. Long-wave elastic anisotropy produced by horizontal layering, Journal of Geophysical Research, 67(11), 4427-4440.

Basu, D., Whittaker, A. S., \& Constantinou, M. C., 2015. Characterizing rotational components of earthquake ground motion using a surface distribution method and response of sample structures, Engineering Structures, 99, 685-707.

Berger, J. \& Beaumont, C., 1976. An analysis of tidal strain observations from the United States of America II. The inhomogeneous tide, Bulletin of the Seismological Society of America, 66(6), 18211846.

Bernauer, F., Wassermann, J., Guattari, F., Frenois, A., Bigueur, A., Gaillot, A., de Toldi, E., Ponceau, D., Schreiber, K. U., \& Igel, H., 2018. BlueSeis3A: Full characterization of a 3C broadband rotational seismometer, Seismological Research Letters, 89(2A), 620-629.

Bernauer, M., Fichtner, A., \& Igel, H., 2012. Measurements of translation, rotation and strain: new approaches to seismic processing and inversion, Journal of Seismology, 16(4), 669-681.

Bernauer, M., Fichtner, A., \& Igel, H., 2014. Reducing nonuniqueness in finite source inversion using rotational ground motions, Journal of Geophysical Research: Solid Earth, 119(6), 4860-4875.

Bodin, P., Gomberg, J., Singh, S. K., \& Santoyo, M., 1997. Dynamic deformations of shallow sediments in the valley of Mexico, part I: Three-dimensional strains and rotations recorded on a seismic array, Bulletin of the Seismological Society of America, 87(3), 528-539.

Brokešová, J. \& Málek, J., 2015a. Six-degree-of-freedom near-source seismic motions I: rotation-totranslation relations and synthetic examples, Journal of Seismology, 19(2), 491-509.

Brokešová, J. \& Málek, J., 2015b. Six-degree-of-freedom near-source seismic motions II: examples of real seismogram analysis and S-wave velocity retrieval, Journal of Seismology, 19(2), 511-539.

Brokešová, J. \& Málek, J., 2013. Rotaphone, a self-calibrated six-degree-of-freedom seismic sensor and its strong-motion records, Seismological Research Letters, 84(5), 737-744.

Capdeville, Y. \& Métivier, L., 2018. Elastic full waveform inversion based on the homogenization method: Theoretical framework and 2-D numerical illustrations, Geophysical Journal International, 213(2), 1093-1112.

Capdeville, Y., Guillot, L., \& Marigo, J.-J., 2010a. 2-D non-periodic homogenization to upscale elastic 
media for P-SV waves, Geophysical Journal International, 182(2), 903-922.

Capdeville, Y., Guillot, L., \& Marigo, J.-J., 2010b. 1-D non-periodic homogenization for the seismic wave equation, Geophysical Journal International, 181(2), 897-910.

Castellani, A. \& Boffi, G., 1989. On the rotational components of seismic motion, Earthquake Engineering \& Structural Dynamics, 18(6), 785-797.

Chapman, C., 2004. Fundamentals of Seismic Wave Propagation, Cambridge University Press.

Cochard, A., Igel, H., Schuberth, B., Suryanto, W., Velikoseltsev, A., Schreiber, K. U., Wassermann, J., Scherbaum, F., \& Vollmer, D., 2006. Rotational motions in seismology: theory, observation, simulation, in Earthquake source asymmetry, structural media and rotation effects, pp. 391-411, Springer-Verlag.

Cupillard, P. \& Capdeville, Y., 2018. Non-periodic homogenization of 3-D elastic media for the seismic wave equation, Geophysical Journal International, 213(2), 983-1001.

Dal Maso, G., 1993. An introduction to $\Gamma$-Convergence, Birkhäuser Basel.

Dean, T., Cuny, T., \& Hartog, A. H., 2017. The effect of gauge length on axially incident P-waves measured using fibre optic distributed vibration sensing, Geophysical Prospecting, 65(1), 184-193.

Donner, S., Bernauer, M., \& Igel, H., 2016. Inversion for seismic moment tensors combining translational and rotational ground motions, Geophysical Journal International, 207(1), 562-570.

Donner, S., Igel, H., Hadziioannou, C., \& the Romy group, 2017. Retrieval of the seismic moment tensor from joint measurements of translational and rotational ground motions: Sparse networks and single stations, in Moment Tensor Solutions, pp. 263-280, Springer International Publishing.

Dziewoński, A. M. \& Anderson, D. L., 1981. Preliminary reference earth model, Physics of the Earth and Planetary Interiors, 25(4), 297-356.

Edme, P. \& Yuan, S., 2016. Local dispersion curve estimation from seismic ambient noise using spatial gradients, Interpretation, 4(3), SJ17-SJ27.

Egorov, E., Egorov, I., \& Agafonov, V., 2015. Self-noise of the MET angular motion seismic sensors, Journal of Sensors, 2015, 1-5.

Ekström, G., Nettles, M., \& Dziewoński, A., 2012. The global CMT project 2004-2010: Centroid-moment tensors for 13,017 earthquakes, Physics of the Earth and Planetary Interiors, 200-201, 1-9.

Fichtner, A. \& Igel, H., 2009. Sensitivity densities for rotational ground-motion measurements, Bulletin of the Seismological Society of America, 99(2B), 1302-1314.

Gomberg, J. \& Agnew, D., 1996. The accuracy of seismic estimates of dynamic strains: An evaluation using strainmeter and seismometer data from Piñon Flat Observatory, California, Bulletin of the Seismological Society of America, 86(1A), 212-220.

Graizer, V., 2009. The response to complex ground motions of seismometers with Galperin sensor configuration, Bulletin of The Seismological Society of America, 99(2B), 1366-1377.

Guillot, L., Capdeville, Y., \& Marigo, J.-J., 2010. 2-D non-periodic homogenization of the elastic wave 

equation: SH case, Geophysical Journal International, 182(3), 1438-1454.

Harrison, J. C., 1976. Cavity and topographic effects in tilt and strain measurement, Journal of Geophysical Research, 81(2), 319-328.

Huang, B.-S., 2003. Ground rotational motions of the 1999 Chi-Chi, Taiwan earthquake as inferred from dense array observations, Geophysical Research Letters, 30(6).

Huang, H., Agafonov, V., \& Yu, H., 2013. Molecular electric transducers as motion sensors: A review, Sensors, 13(4), 4581-4597.

Igel, H., Schreiber, K. U., Flaws, A., Schuberth, B., Velikoseltsev, A., \& Cochard, A., 2005. Rotational motions induced by the M8.1 Tokachi-oki earthquake, September 25, 2003, Geophysical Research Letters, 32(8).

Igel, H., Cochard, A., Wassermann, J., Flaws, A., Schreiber, K. U., Velikoseltsev, A., \& Pham Dinh, N., 2007. Broad-band observations of earthquake-induced rotational ground motions, Geophysical Journal International, 168(1), 182-196.

Igel, H., Bernauer, M., Wassermann, J., \& Schreiber, K. U., 2015. Rotational seismology: Theory, instrumentation, observations, applications, in Encyclopedia of Complexity and Systems Science, SpringerVerlag New York.

King, G. \& Bilham, R., 1973. Tidal tilt measurement in europe, Nature, 243(5402), 74-75.

Kohl, M. L. \& Levine, J., 1995. Measurement and interpretation of tidal tilts in a small array, Journal of Geophysical Research, 100(B3), 3929-3941.

Komatitsch, D. \& Vilotte, J. P., 1998. The spectral-element method: An efficient tool to simulate the seismic response of 2D and 3D geological structures, Bulletin of the Seismological Society of America, 88(2), 368-392.

Krischer, L., Megies, T., Barsch, R., Beyreuther, M., Lecocq, T., Caudron, C., \& Wassermann, J., 2015. Obspy : a bridge for seismology into the scientific Python ecosystem, Computational Science \& Discovery, 8(1).

Kurzych, A., Jaroszewicz, L. R., Krajewski, Z., Teisseyre, K. P., \& Kowalski, J. K., 2014. Fibre optic system for monitoring rotational seismic phenomena, Sensors, 14(3), 5459-5469.

Langston, C. A., 2007. Wave gradiometry in two dimensions, Bulletin of the Seismological Society of America, 97(2), 401-416.

Lennon, G. \& Baker, T., 1973. A discussion on the measurement and interpretation of changes of strain in the Earth - Earth tides and their place in geophysics (summary), Philosophical Transactions of the Royal Society of London. Series A, Mathematical and Physical Sciences, 274(1239), 199-202.

Li, Z. \& van der Baan, M., 2017. Tutorial on rotational seismology and its applications in exploration geophysics, Geophysics, 82(5), W17-W30.

Lindner, F., Wassermann, J., Schmidt-Aursch, M. C., Schreiber, K. U., \& Igel, H., 2016. Seafloor ground 
rotation observations: Potential for improving signal-to-noise ratio on horizontal OBS components, Seismological Research Letters, 88(1), 32-38.

McLeod, D. P., Stedman, G. E., Webb, T. H., \& Schreiber, K. U., 1998. Comparison of standard and ring laser rotational seismograms, Bulletin of the Seismological Society of America, 88(6), 1495-1503.

Mikumo, T. \& Aki, K., 1964. Determination of local phase velocity by intercomparison of seismograms from strain and pendulum instruments, Journal of Geophysical Research, 69(4), 721-731.

Pancha, A., Webb, T. H., Stedman, G. E., McLeod, D. P., \& Schreiber, K. U., 2000. Ring laser detection of rotations from teleseismic waves, Geophysical Research Letters, 27(21), 3553-3556.

Pierson, B., Laughlin, D., \& Brune, R., 2016. Advances in rotational seismic measurements, in SEG Technical Program Expanded Abstracts 2016, pp. 2263-2267, Society of Exploration Geophysicists.

Poppeliers, C. \& Evans, E. V., 2015. The effects of measurement uncertainties in seismic-wave gradiometry, Bulletin of the Seismological Society of America, 105(6), 3143-3155.

Reinwald, M., Bernauer, M., Igel, H., \& Donner, S., 2016. Improved finite-source inversion through joint measurements of rotational and translational ground motions: a numerical study, Solid Earth, 7(5), 14671477.

Schmelzbach, C., Donner, S., Igel, H., Sollberger, D., Taufiqurrahman, T., Bernauer, F., Häusler, M., Renterghem, C. V., Wassermann, J., \& Robertsson, J., 2018. Advances in 6C seismology: Applications of combined translational and rotational motion measurements in global and exploration seismology, Geophysics, 83(3), WC53-WC69.

Schreiber, K. U., Velikoseltsev, A., Igel, H., Cochard, A., Flaws, A., Drewitz, W., \& Müller, F., 2003. The geosensor: A new instrument for seismology, in Geotechnologien Science Report No. 3: "Observation of the System Earth from Space”, Status Seminar, Bavarian State Mapping Agency (BLVA), Munich, 12-13 June 2003, Programme \& Abstracts.

Schreiber, K. U., Hautmann, J. N., Velikoseltsev, A., Wassermann, J., Igel, H., Otero, J., Vernon, F., \& Wells, J. P. R., 2009. Ring laser measurements of ground rotations for seismology, Bulletin of the Seismological Society of America, 99(2B), 1190-1198.

Sollberger, D., Schmelzbach, C., Renterghem, C. V., Robertsson, J., \& Greenhalgh, S., 2016. Singlecomponent elastic wavefield separation at the free surface using source- and receiver-side gradients, in

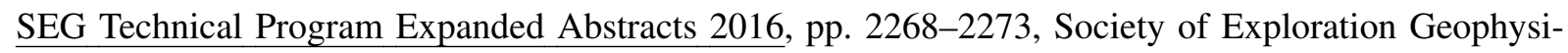
cists.

Spudich, P., Steck, L. K., Hellweg, M., Fletcher, J. B., \& Baker, L. M., 1995. Transient stresses at Parkfield, California, produced by the M7.4 Landers earthquake of June 28, 1992: Observations from the UPSAR dense seismograph array, Journal of Geophysical Research: Solid Earth, 100(B1), 675-690.

Suryanto, W., Igel, H., Wassermann, J., Cochard, A., Schuberth, B., Vollmer, D., Scherbaum, F., Schreiber, K. U., \& Velikoseltsev, A., 2006. First comparison of array-derived rotational ground motions with direct 
ring laser measurements, Bulletin of the Seismological Society of America, 96(6), 2059-2071.

Takeo, M. \& Ito, H. M., 1997. What can be learned from rotational motions excited by earthquakes?, Geophysical Journal International, 129(2), 319-329.

Tarantola, A. \& Valette, B., 1982. Generalized nonlinear inverse problems solved using the least squares criterion, Reviews of Geophysics, 20(2), 219-232.

Trifunac, M. D., 1982. A note on rotational components of earthquake motions on ground surface for incident body waves, International Journal of Soil Dynamics and Earthquake Engineering, 1(1), 11-19.

Trifunac, M. D. \& Todorovska, M. I., 2001. A note on the useable dynamic range of accelerographs recording translation, Soil Dynamics and Earthquake Engineering, 21(4), 275-286.

van Driel, M., Wassermann, J., Nader, M. F., Schuberth, B. S. A., \& Igel, H., 2012. Strain rotation coupling and its implications on the measurement of rotational ground motions, Journal of Seismology, 16(4), $657-668$.

Wassermann, J., Wietek, A., Hadziioannou, C., \& Igel, H., 2016. Toward a single-station approach for microzonation: Using vertical rotation rate to estimate Love-wave dispersion curves and direction finding, Bulletin of the Seismological Society of America, 106(3), 1316-1330.

Willis, M. E., Barfoot, D., Ellmauthaler, A., Wu, X., Barrios, O., Erdemir, C., Shaw, S., \& Quinn, D., 2016. Quantitative quality of distributed acoustic sensing vertical seismic profile data, The Leading Edge, 35(7), 605-609.

\section{APPENDIX A: 2D NON-PERIODIC HOMOGENIZATION}

Here, we give a brief overview on the theory of homogenization for the $2 \mathrm{D}$ case. For a more comprehensive description, Capdeville et al. (2010b), Capdeville et al. (2010a) and Guillot et al. (2010), Cupillard \& Capdeville (2018) are proposed for the 1D, 2D and 3D case respectively.

Homogenization deals with the obtaining homogenized or effective equations for systems with fine microscopic structure. The limit between the macroscopic and microscopic variables is denoted by $\varepsilon=\frac{\lambda_{0}}{\lambda_{m}}$, where $\lambda_{0}$ is the length-scale below which scales are considered microscopic and $\lambda_{m}$ is the minimum wavelength in the medium.

To separate the microscopic and macroscopic variables explicitly, a new space variable $\mathbf{y}=$ $\frac{\mathrm{x}}{\varepsilon}$ is introduced such that $\mathbf{x}$ encapsulates all the macroscopic variations and $\mathbf{y}$ encapsulates all the microscopic variations. It is assumed that $\mathbf{x}$ and $\mathbf{y}$ can be treated as independent variables. 
Consequently, all partial derivatives with respect to $\mathrm{x}$ are redefined as

$$
\nabla_{\mathbf{x}} \rightarrow \nabla_{\mathbf{x}}+\frac{1}{\varepsilon} \nabla_{\mathbf{y}}
$$

In homogenization, displacement $\mathbf{u}(\mathbf{x}, t)$ and stress $\boldsymbol{\sigma}(\mathbf{x}, t)$ are postulated to be two-scale asymptotic expansions in $\varepsilon$ :

$$
\begin{aligned}
& \mathbf{u}^{\varepsilon}(\mathbf{x}, t)=\sum_{i=0}^{\infty} \varepsilon^{i} \mathbf{u}^{i}(\mathbf{x}, \mathbf{y}, t), \\
& \boldsymbol{\sigma}^{\varepsilon}(\mathbf{x}, t)=\sum_{i=-1}^{\infty} \varepsilon^{i} \boldsymbol{\sigma}^{i}(\mathbf{x}, \mathbf{y}, t),
\end{aligned}
$$

where the $i$ are powers in $\varepsilon$ but not on the $\mathbf{u}$ and $\boldsymbol{\sigma}$. Substituting Equations (A.1) and (A.2) into the wave equation yields a series of equations which can be solved for each $i$. For $i=0$, it emerges that $\mathbf{u}^{0}$ does not depend on the microscopic variable $\mathbf{y}$, i.e. to the leading order,

$$
\mathbf{u}^{\varepsilon}(\mathbf{x}, \mathbf{y}, t)=\mathbf{u}^{0}(\mathbf{x}, t)
$$

Unlike $i=0$, the higher order terms in the series (A.2) do depend on $\mathbf{y}$, for example,

$$
\mathbf{u}^{1}(\mathbf{x}, \mathbf{y}, t)=\chi^{1}(\mathbf{x}, \mathbf{y}, t) \boldsymbol{\epsilon}_{\mathbf{x}}\left(\mathbf{u}^{0}(\mathbf{x}, t)\right)+\left\langle\mathbf{u}^{1}(\mathbf{x}, t)\right\rangle
$$

where $\chi^{1}$ is the first-order corrector, a third-order tensor. Going further, we also obtain the equation of the cell problem:

$$
\nabla_{\mathbf{y}}\left(\mathbf{c}^{\varepsilon} \cdot\left(\mathbf{I}+\nabla_{\mathbf{y}} \chi^{1}\right)\right)=0
$$

where $\mathbf{c}$ is the elastic tensor.

In fact, $\mathbf{u}^{0}$, the zeroth order effective displacement, is a solution of the classical wave equation

$$
\rho^{*} \ddot{\mathbf{u}}^{0}-\nabla \cdot \mathbf{c}^{*}: \nabla \mathbf{u}^{0}=\boldsymbol{f}^{*}
$$

where the effective model $\left(\rho^{*}, \mathbf{c}^{*}\right)$ can be obtained by solving the cell problem (Capdeville et al. 2010a).

The corresponding equations for gradients can be derived using (A.1), (A.3) and (A.4). 


\section{APPENDIX B: CORRECTOR FOR ARRAY-DERIVED WAVEFIELD GRADIENT}

It is possible to estimate wavefield gradients just from displacements. The most straightforward procedure is based on truncated Taylor series,

$$
h \nabla_{\mathbf{j}} f(\mathbf{x}) \sim f(\mathbf{x}+h \mathbf{j})-f(\mathbf{x}),
$$

where $\mathbf{j} \in\left\{\hat{\mathbf{x}}_{\mathbf{1}}, \hat{\mathbf{x}}_{\mathbf{2}}\right\}$. This approximation is correct to first-order in grid spacing $h$ and is only valid if $f$ is smooth with respect to $h$. Recalling the equation for true displacement from (7),

$$
\mathbf{u}^{\varepsilon}(\mathbf{x}, \mathbf{y}, t)=\mathbf{u}^{0}(\mathbf{x}, t)+\varepsilon\left(\chi(\mathbf{y}): \boldsymbol{\epsilon}\left(\mathbf{u}^{0}(\mathbf{x}, t)\right)\right) .
$$

Expanding the terms in (B.2) individually, noting that the corrector $\chi$ is not smooth with respect to $\mathbf{x}$, and using the relation $\mathbf{y}=\frac{\mathbf{x}}{\varepsilon}$, we have,

$$
\mathbf{u}^{\varepsilon}\left(\mathbf{x}+h \mathbf{j}, \mathbf{y}+\frac{h}{\varepsilon} \mathbf{j}, t\right)=\mathbf{u}^{0}(\mathbf{x}, t)+h \nabla_{\mathbf{j}} \mathbf{u}^{0}(\mathbf{x}, t)+\varepsilon\left(\chi\left(\mathbf{y}+\frac{h}{\varepsilon} \mathbf{j}\right): \boldsymbol{\epsilon}\left(\mathbf{u}^{0}(\mathbf{x}, t)+h \nabla_{\mathbf{j}} \mathbf{u}^{0}(\mathbf{x}, t)\right)\right) .
$$

Substituting (B.2) and (B.3) in (B.1), we get,

$$
\begin{aligned}
\mathbf{u}^{\varepsilon}\left(\mathbf{x}+h \mathbf{j}, \mathbf{y}+\frac{h}{\varepsilon} \mathbf{j}, t\right)-\mathbf{u}^{\varepsilon}(\mathbf{x}, \mathbf{y}, t) & =h \nabla_{\mathbf{j}} \mathbf{u}^{0}(\mathbf{x}, t)+\varepsilon\left(\chi\left(\mathbf{y}+\frac{h}{\varepsilon} \mathbf{j}\right)-\chi(\mathbf{y})\right): \boldsymbol{\epsilon}\left(\mathbf{u}^{0}(\mathbf{x}, t)\right), \\
\frac{\mathbf{u}^{\varepsilon}\left(\mathbf{x}+h \mathbf{j}, \mathbf{y}+\frac{h}{\varepsilon} \mathbf{j}, t\right)-\mathbf{u}^{\varepsilon}(\mathbf{x}, \mathbf{y}, t)}{h} & =\nabla_{\mathbf{j}} \mathbf{u}^{0}(\mathbf{x}, t)+\mathbf{J}(\mathbf{y}): \boldsymbol{\epsilon}\left(\mathbf{u}^{0}(\mathbf{x}, t)\right) \\
\nabla_{\mathbf{j}} \mathbf{u}^{\varepsilon}(\mathbf{x}, \mathbf{y}, t) & =\nabla_{\mathbf{j}} \mathbf{u}^{0}(\mathbf{x}, t)+\mathbf{J}(\mathbf{y}): \boldsymbol{\epsilon}\left(\mathbf{u}^{0}(\mathbf{x}, t)\right)
\end{aligned}
$$

with $\mathbf{J}(\mathbf{y})=\frac{\varepsilon}{h}\left(\chi\left(\mathbf{y}+\frac{h}{\varepsilon} \mathbf{j}\right)-\chi(\mathbf{y})\right)$.

\section{APPENDIX C: TRANSLATIONS ARE IN GOOD AGREEMENT}

As stated in section 2.3 , the effective model needed for the inversion of $\mathbf{J}$ is not limited to the homogenized model. Any tomographic model that fits displacement data reasonably well can be used.

In section 3.1, we used the homogeneous medium as our effective model and it is indeed sensible to do so because as shown by Figure A1, the displacements in both the heterogeneous and homogeneous media are in agreement. This reasoning is also extendable to inversion in section 


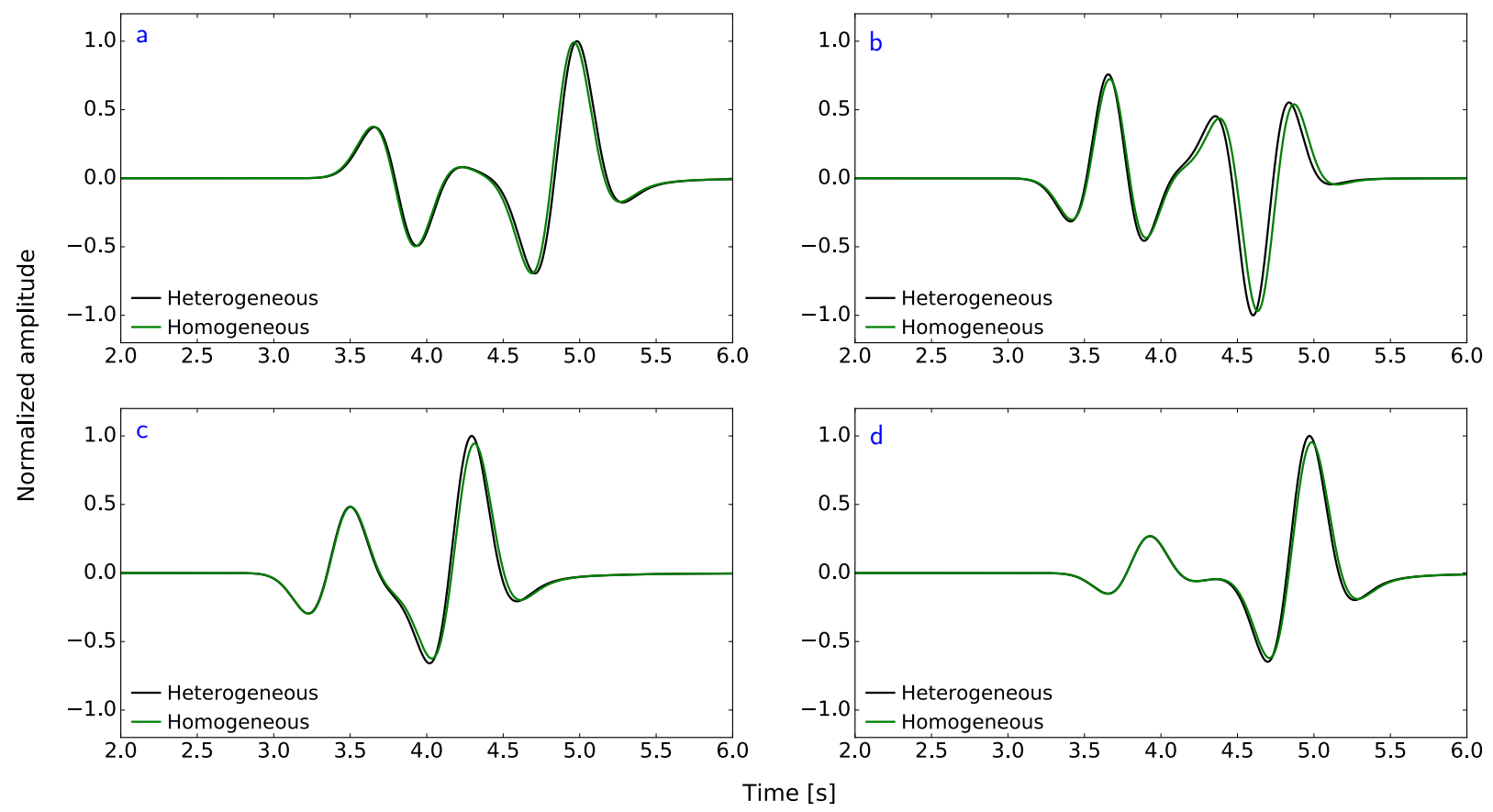

Figure A1. Vertical displacements for the four events shown in Figure 4. Displacements in the heterogeneous media are shown in black and the displacements in the homogeneous media are shown in green.

Similarly, in section 4.1, we perform the inversion for $\mathbf{J}$ for the ring laser at Wettzell, Germany. In this case, our effective model was PREM. Figure A2 shows that displacement data at the collocated seismometer WET and synthetics derived in this effective model are in agreement. Therefore, the use of PREM here is justifiable. Note that the time axis are different than in Figure 7 to adjust the Rayleigh wave arrivals.

In section 4.2, we estimated array derived rotation rates at the ring laser. From Figure A3, we see that the velocities at three stations are in agreement. The distance between the stations is around $100 \mathrm{~km}$.

\section{APPENDIX D: SOURCE-RECEIVER DISTRIBUTION}

Here, we show the map of the earthquakes and station data set used in 4. Figure A4 shows the source-receiver distribution for the inversion in 4.1, while Figure A5 shows the source-receiver distribution for the inversion in 4.2. 


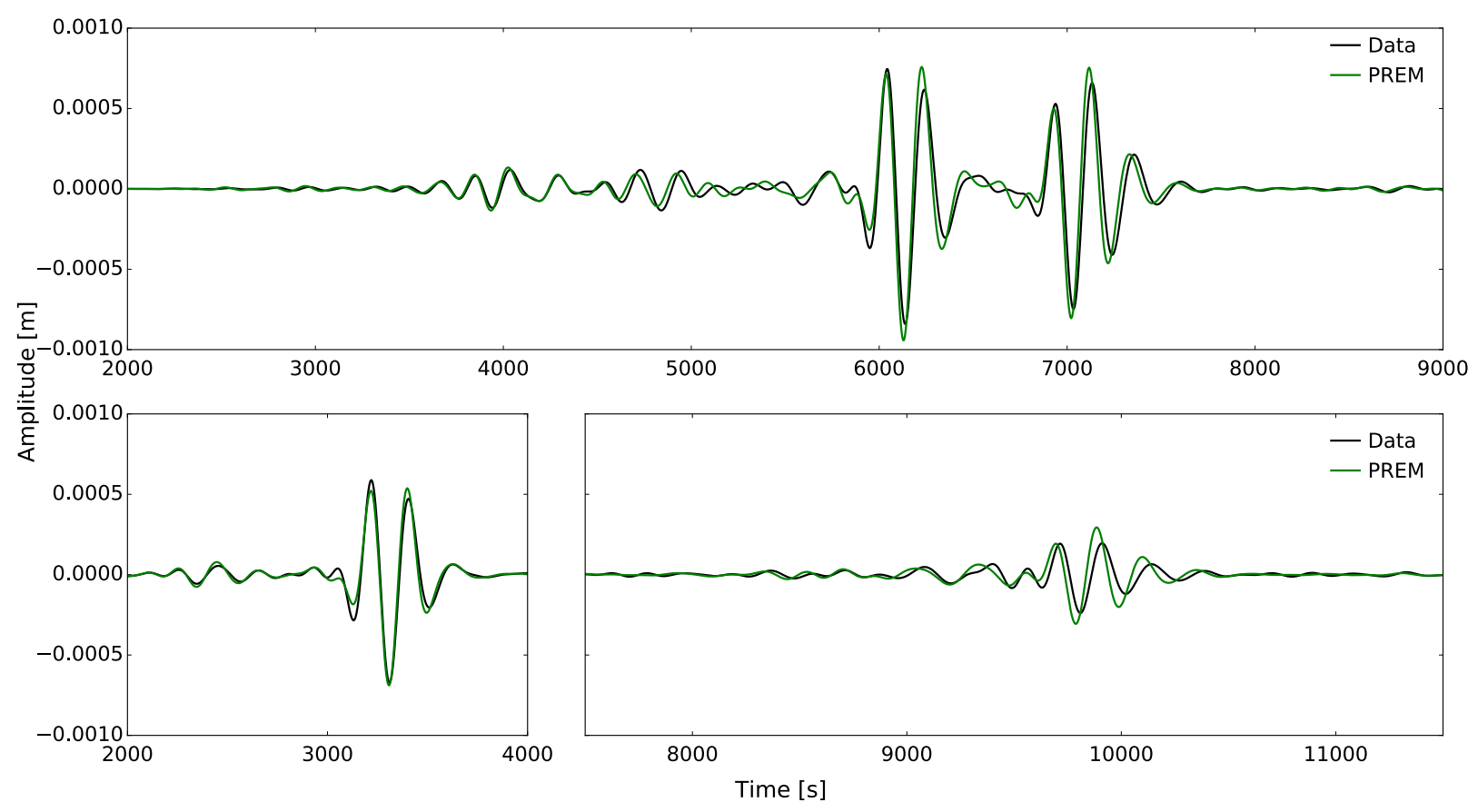

Figure A2. Vertical displacements measured at WET for the two events shown in Figure 7. Observed displacements are shown in black and the displacements computed in PREM are shown in green.

Top: 201611131102A South Island, New Zealand. Bottom: 201210280304A Queen Charlotte Islands.

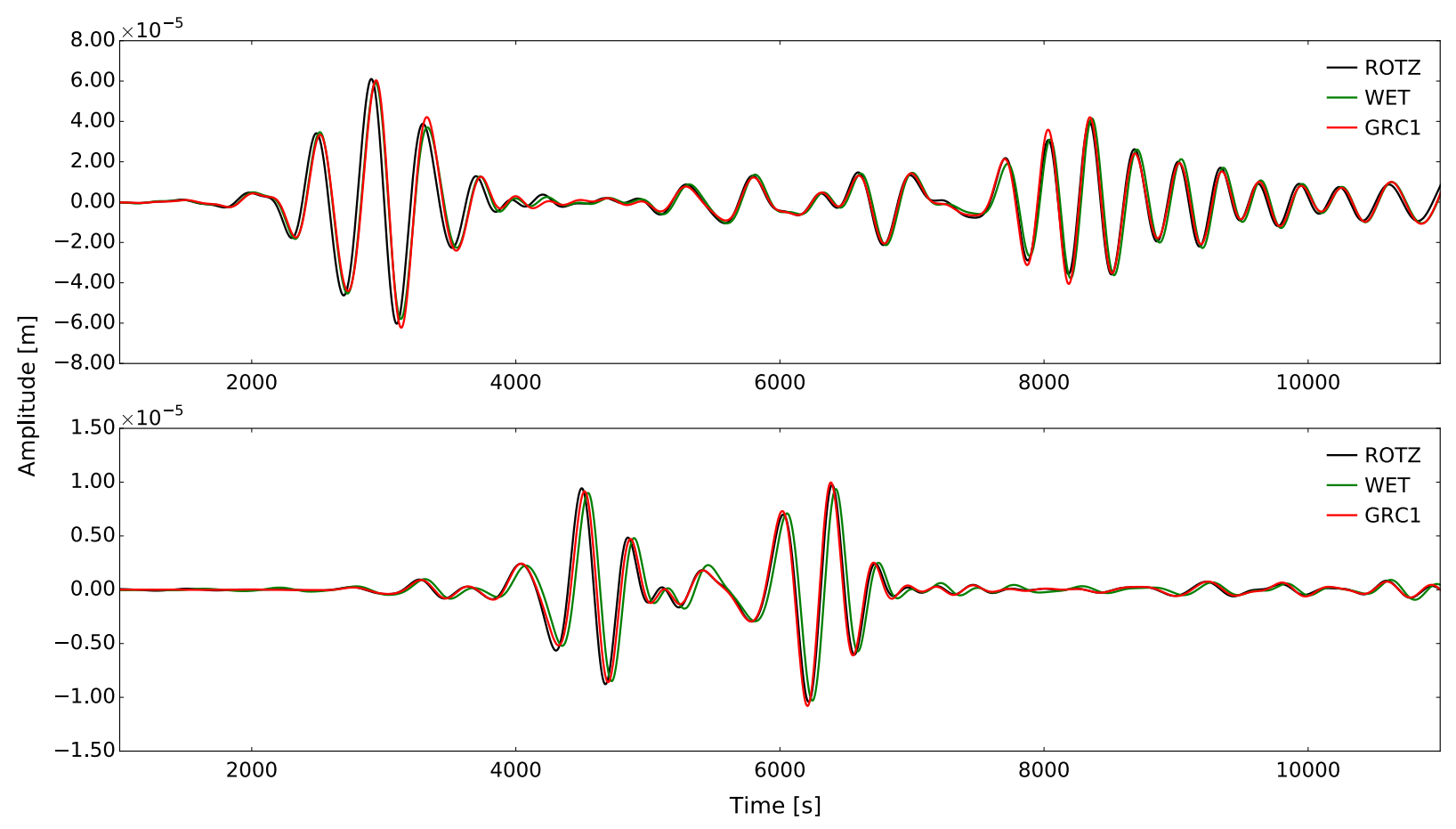

Figure A3. Velocities along the East component for the two events shown in Figure 9. Observed velocities at stations ROTZ, WET and GRC1 are shown in black, green and red respectively.

Top: 201103110546A Near East Coast of Honsh. Bottom: 200909291748A Samoa Islands Region. 


\section{4}

Singh et al.

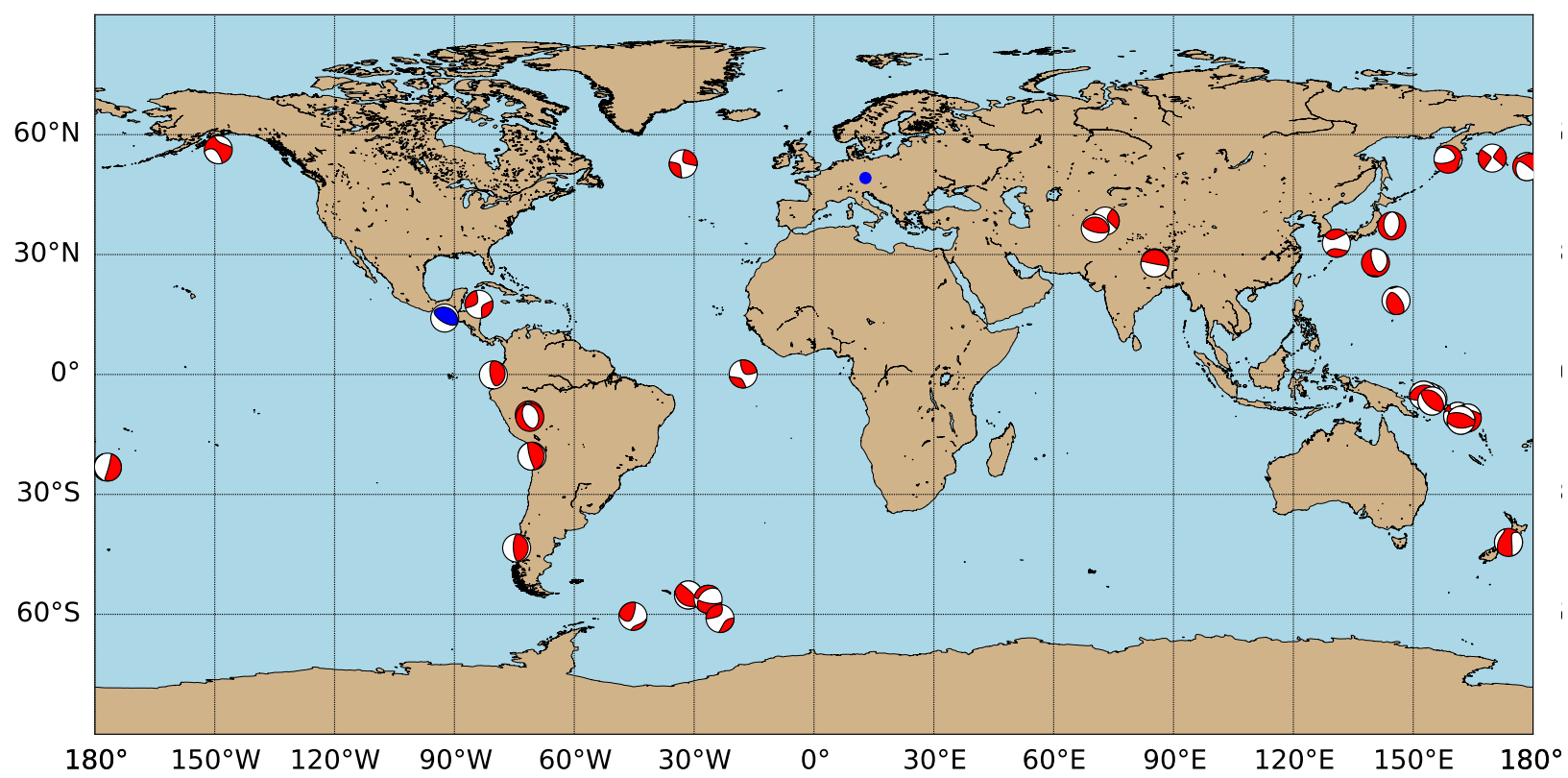

Figure A4. The source receiver geometry for the events in Section 4.1. The 32 events used for the inversion are shown in red, while the event that was used for validation is shown in blue. The blue dot shows the location of the receiver.

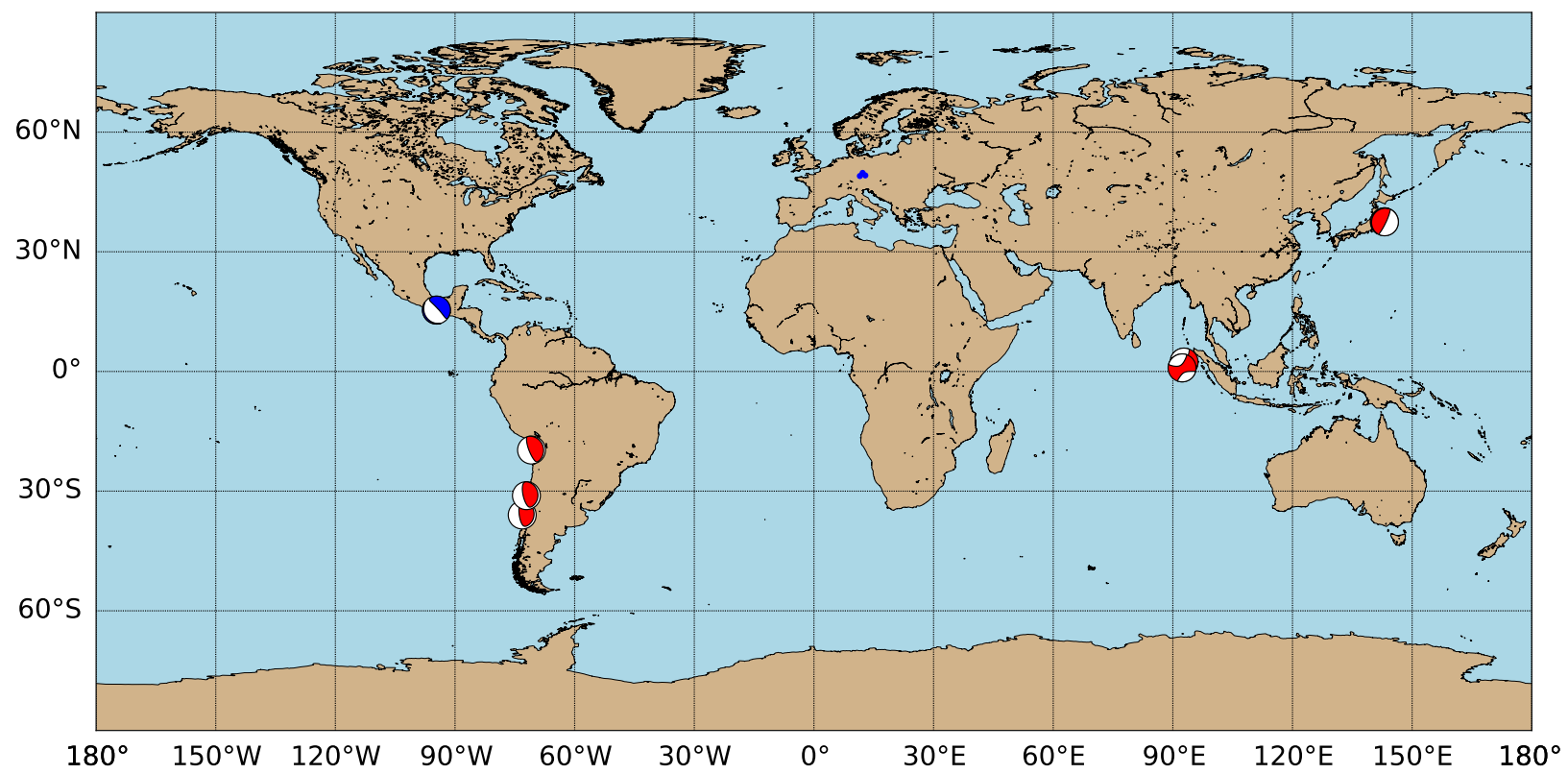

Figure A5. The source receiver geometry for the events in Section 4.2. The 6 events used for the inversion are shown in red, while the event that was used for validation is shown in blue. The blue dots show the location of the three receivers. 